\title{
Duality and an exact Landau-Ginzburg potential for quasi-bosonic Chern-Simons-Matter theories
}

\author{
Anshuman Dey, ${ }^{a}$ Indranil Halder, ${ }^{a}$ Sachin Jain, ${ }^{b}$ Lavneet Janagal, ${ }^{a}$ Shiraz Minwalla ${ }^{a}$ \\ and Naveen Prabhakar ${ }^{a}$ \\ ${ }^{a}$ Department of Theoretical Physics, Tata Institute of Fundamental Research, \\ Homi Bhabha Rd, Mumbai 400005, India \\ ${ }^{b}$ Indian Institute of Science Education and Research, \\ Homi Bhabha Rd, Pashan, Pune 411 008, India \\ E-mail: anshuman@theory.tifr.res.in, indranil.halder@tifr.res.in, \\ sachin.jain@iiserpune.ac.in, lavneet@theory.tifr.res.in, \\ minwalla@theory.tifr.res.in, naveensp@theory.tifr.res.in
}

ABSTRACT: It has been conjectured that Chern-Simons (CS) gauged 'regular' bosons in the fundamental representation are 'level-rank' dual to CS gauged critical fermions also in the fundamental representation. Generic relevant deformations of these conformal field theories lead to one of two distinct massive phases. In previous work, the large $N$ thermal free energy for the bosonic theory in the unHiggsed phase has been demonstrated to match the corresponding fermionic results under duality. In this note we evaluate the large $N$ thermal free energy of the bosonic theory in the Higgsed phase and demonstrate that our results, again, perfectly match the predictions of duality. Our computation is performed in a unitary gauge by integrating out the physical excitations of the theory - i.e. W bosons at all orders in the 't Hooft coupling. Our results allow us to construct an exact quantum effective potential for $\bar{\phi} \phi$, the lightest gauge invariant scalar operator in the theory. In the zero temperature limit this exact Landau-Ginzburg potential is non-analytic at $\bar{\phi} \phi=0$. The extrema of this effective potential at positive $\bar{\phi} \phi$ solve the gap equations in the Higgsed phase while the extrema at negative $\bar{\phi} \phi$ solve the gap equations in the unHiggsed phase. Our effective potential is bounded from below only for a certain range of $x_{6}$ (the parameter that governs sextic interactions of $\phi$ ). This observation suggests that the regular boson theory has a stable vacuum only when $x_{6}$ lies in this range.

Keywords: 1/N Expansion, Chern-Simons Theories, Duality in Gauge Field Theories, Renormalization Group

ArXiv EPrint: 1808.04415 


\section{Contents}

1 Introduction 2

2 Review of known results 5

2.1 Theories and the conjectured duality map 5

$\begin{array}{ll}2.2 \text { Structure of the thermal partition function } & 6\end{array}$

3 The Higgsed phase of the regular boson theory $\quad 8$

3.1 Lagrangian in unitary gauge 8

$\begin{array}{llr}3.2 & \text { An effective action for the Higgs field } V & 9\end{array}$

$\begin{array}{lll}3.3 & \text { Tadpole cancellation for } V & 12\end{array}$

4 A three variable off-shell free energy $\quad 14$

5 The exact Landau-Ginzburg effective potential $\quad \mathbf{1 6}$

$\begin{array}{ll}5.1 \text { An effective potential for } \sigma_{B} & 16\end{array}$

$\begin{array}{ll}5.2 \text { Higgsed branch } & 19\end{array}$

$\begin{array}{lll}\text { 5.2.1 Potential for the Higgs vev } & 19\end{array}$

$\begin{array}{ll}\text { 5.2.2 Graphs of } U_{\text {eff }}\left(\sigma_{B}\right) \text { in various cases } & 19\end{array}$

$\begin{array}{ll}5.3 \text { unHiggsed branch } & 21\end{array}$

$\begin{array}{lll}\text { 5.3.1 Graphs of } U_{\text {eff }}\left(\sigma_{B}\right) \text { in various cases } & 21\end{array}$

5.3.2 Explanation for the instability of local maxima 23

5.4 Landau-Ginzburg analysis of the zero temperature phase diagram 25

$\begin{array}{lll}\text { 5.4.1 Case I: } x_{6}>\phi_{2} & 26\end{array}$

5.4.2 Case II: $\phi_{1}<x_{6}<\phi_{2} \operatorname{phi}(1)=\mathrm{x}(6)=\operatorname{phi}(2) \quad 27$

$\begin{array}{lll}5.4 .3 & \text { Case III: } x_{6}<\phi_{1}=\operatorname{phi}(1) & 29\end{array}$

6 Discussion 31

A Previously known results for the large $N$ free energy $\quad 32$

A.1 Results for the critical fermion theory 32

A.2 Results for regular bosons in the unHiggsed phase 34

A.3 Duality in the unHiggsed phase and a prediction for the Higgsed phase $\quad 35$

A.3.1 unHiggsed phase: $\operatorname{sgn}\left(X_{F}\right)=-\operatorname{sgn}\left(\lambda_{B}\right) \quad 36$

$\begin{array}{lll}\text { A.3.2 Higgsed phase: } \operatorname{sgn}\left(X_{F}\right)=\operatorname{sgn}\left(\lambda_{B}\right) & 37\end{array}$

$\begin{array}{ll}\text { B The tadpole from } W \text { boson loops } & 37\end{array}$

$\begin{array}{ll}\text { C The critical boson scaling limit } & 41\end{array}$ 


\section{Introduction}

There is now considerable evidence that a single fermionic field in the fundamental of $\mathrm{U}\left(N_{F}\right)$ minimally coupled to $\mathrm{U}\left(N_{F}\right)$ Chern-Simons gauge theory at level ${ }^{1} k_{F}$ is dual to vector $\mathrm{SU}\left(N_{B}\right)$ Wilson-Fisher scalars minimally coupled to $\mathrm{SU}\left(N_{B}\right)$ Chern-Simons gauge theory at level $k_{B}=-\operatorname{sgn}\left(k_{F}\right) N_{F}$ with $N_{B}=\left|k_{F}\right|$ [1]-[70]. ${ }^{2}$ This (almost standard by now) duality asserts that the two so-called quasi-fermionic CFTs i.e. Chern-Simons gauged 'regular fermions' (RF) and 'critical bosons' (CB) - are secretly the same theory.

It has also been conjectured (see [2] and references therein) that the 'quasi-fermionic' duality of the previous paragraph follows as the infrared limit of a duality between pairs of fermionic and bosonic RG flows. The fermionic RG flows are obtained by starting in the ultraviolet with the Chern-Simons gauged Gross-Neveu or 'critical fermion' (CF) theory and deforming this theory with relevant operators fine tuned to ensure that the IR end point of the RG flow is the RF theory. In a similar manner the conjecturally dual bosonic flows are obtained by starting in the ultraviolet with the gauged 'regular boson' (RB) theory deformed with the fine tuning that ensures that the RG flow ends in the CB theory.

The UV starting points of the flows described above define dual pairs of conformal field theories. These RB and CF theories - so-called quasi-bosonic theories - are conjectured to be dual to each other. ${ }^{3}$ If valid, this conjecture implies that the set of all RG flows that originate in the $\mathrm{RB}$ theory are dual to the set of all $\mathrm{RG}$ flows that originate in the $\mathrm{CF}$ theory. The duality of the pair of specially tuned RG flows of the last paragraph- those that end in the IR in the quasi-fermionic conformal field theories - is a special case of this general phenomenon.

Generic RG flows that originate at quasi-bosonic fixed points lead to gapped phases, or more accurately, phases whose low energy behaviour is governed by a topological field theory. There are two inequivalent topological phases. In the unHiggsed phase the bosonic (resp. fermionic) theory is governed at long distances by pure $\mathrm{SU}\left(N_{B}\right)_{k_{B}}$ (resp. $\mathrm{U}\left(N_{F}\right)_{k_{F}}$ ) topological field theory with the two theories being level-rank dual to each other. In the Higgsed phase the bosonic (resp. fermionic) theory is governed in the IR by a pure $\mathrm{SU}\left(N_{B}-1\right)_{k_{B}}$ (resp. $\left.\mathrm{U}\left(N_{F}\right)_{\operatorname{sgn} k_{F}\left(\left|k_{F}\right|-1\right)}\right)$ Chern-Simons theory with the two topological field theories once again being level-rank dual to each other. ${ }^{4}$

\footnotetext{
${ }^{1}$ In our conventions the level of a Chern-Simons theory coupled to fermions is defined to be the level of the low energy gauge group obtained after deforming the theory with a fermion mass of the same sign as the fermion level.

${ }^{2}$ See the introduction to the recent paper [1] for a more more detailed description of earlier work.

${ }^{3}$ At leading order in large $N$ - the order to which we work in this paper - the RB and CF theories appear as a line of fixed points parametrized by the single parameter $x_{6}$, the coefficient of the $\phi^{6}$ coupling of the bosonic theory (see below for the dual statement in the fermionic theory). In other words the one parameter set of RB and CF theories (and flows originating therein) that we study in this paper are actually only physical at three particular values of the parameter $x_{6}$. See the very recent paper [3] for a computation of the beta function for $x_{6}$ that establishes this point.

${ }^{4}$ As first explained in [4], the reduction in rank of the bosonic Chern-Simons theory compared to the unHiggsed phase is a consequence of the Higgs mechanism in the bosonic field theory. The reduction in level of the fermionic Chern-Simons theory is a consequence of the switch in sign of the mass of the fermion - level of the pure Chern-Simons theory obtained by integrating out a negative mass fermion is one unit smaller than the level obtained by integrating out a positive mass fermion.
} 
The most compelling evidence for the scenarios spelt out above comes from explicit results of direct all-orders calculations that have been performed separately in the fermionic and bosonic theories in the large $N$ limit. In particular, the thermal partition function of deformed RF and $\mathrm{CB}$ theories have both been computed in the unHiggsed phases to all orders in the 't Hooft coupling, and have been shown to match exactly with each other for all relevant deformations that end up in this phase [2]. ${ }^{5}$ While impressive, this matching is incomplete, as the restriction to the unHiggsed phase covers only half of the phase diagram of these theories.

The authors of [2] (and references therein) were also able to compute the thermal partition function of the CF theory in the 'Higgsed' phase. However, they were unable to perform the analogous computation in the bosonic theory in this phase and so were unable to verify the matching of thermal free energies in this phase. In this paper we fill the gap described above. We present an explicit all-orders computation of the thermal free energy of the RB theory in the Higgsed phase. Under duality our final results exactly match the free energy of the fermionic theory in the Higgsed phase, completing the large $N$ check of the conjectured duality in a satisfying manner.

At the technical level, the computation described in the previous paragraph (and presented in detail in section 3) is a relatively straightforward generalisation of the computations presented in the recent paper [1]. In [1] the large $N$ free energy of the Higgsed phase of the Chern-Simons gauged Wilson-Fisher boson theory was computed for the first time. As we describe in much more detail below, the computation of the free energy in the Higgsed phase of the RB theory can be divided into two steps. In the first step we compute the thermal free energy (or equivalently, the gap equation) of the CB theory as a function of its Higgs vev. We are able to import this computation directly from [1]. In the relatively simple second step carried through in this paper, we derive a second gap equation that determines the effective value of the Higgs vev.

The second step described at the end of the last paragraph had no counterpart in [1]. In the critical bosonic theory the 'classical' potential for the scalar field is infinitely deep. This potential freezes the magnitude of the scalar field in the Higgsed phase to its classical minimum even in the quantum theory. It follows that the Higgs vev is independent of the temperature and has a simple dependence on the 't Hooft coupling in the critical boson theory. In the regular boson theory, on the other hand, the classical potential for the scalar field is finite and receives nontrivial quantum corrections. The value of the scalar condensate is determined extremizing the quantum effective action for the scalar field. The result of this minimisation yields a scalar vev that is a nontrivial function of both the 't Hooft coupling and the temperature. It follows that the computations of this paper give us a bonus: we are able to compute the smooth 'quantum effective potential' for the RB theory as a function of the Higgs vev. More precisely we compute the quantum effective potential for the composite field $(\bar{\phi} \phi)$. In the Higgsed phase and in the unitary gauge employed in the computations of this paper, this quantity reduces to a potential — an

\footnotetext{
${ }^{5}$ A similar matching has also been performed for the S-matrix in the unHiggsed phase [5, 6]. The generalisation of this match to the Higgsed phase is also an interesting project, but one that we will not consider in this paper.
} 
exact Landau-Ginzburg effective potential - for the Higgs vev. The extremization of this potential determines the Higgs vev - and is an equivalent and intuitively satisfying way of obtaining the gap equations - in the Higgsed phase. The later sections of this paper sections 4 and 5 are devoted to the study of the exact quantum effective potential of the theory and its physical consequences.

Let us denote the expectation value of $(\bar{\phi} \phi)$ by $(\bar{\phi} \phi)_{\mathrm{cl}}$. At zero temperature it turns out that the quantum effective action is non-analytic at $(\bar{\phi} \phi)_{\mathrm{cl}}=0$. For this reason the domain of the variable in our effective potential - namely $\bar{\phi} \phi$ - naturally splits into two regions. We refer to the region $\bar{\phi} \phi>0$ as the Higgsed branch of our effective potential. On the other hand the region $\bar{\phi} \phi<0$ is the unHiggsed branch of our effective potential. On the Higgsed branch the quantum effective potential for $\bar{\phi} \phi$ is simply a quantum corrected version of the classical potential of the theory. Classically $\bar{\phi} \phi$ always positive, and so the potential for the theory on the unHiggsed branch (i.e. at negative $\bar{\phi} \phi$ ) has no simple classical limit and is purely quantum in nature. The extremization of the effective potential on the Higgsed/unHiggsed branches exactly reproduces the gap equations in the Higgsed/unHiggsed phases.

In both phases the extrema of this effective potential are of two sorts; local maxima and local minima. Local maxima clearly describe unstable 'phases'. The instability of these phases has an obvious semiclassical explanation in the Higgsed phase; it is a consequence of the fact that we have chosen to expand about a maximum of the potential for the Higgs vev. In this paper we find an analogous physical explanation for the instability of the 'maxima' in the unHiggsed phase. In section 5.3 we use the results for exact S-matrices in these theories $[5,6]$ to demonstrate that the 'phases' constructed about maxima in the unHiggsed branch always have bound states of one fundamental particle (created by $\phi$ ) and one antifundamental particle (created by $\bar{\phi}$ ) in the so called 'singlet' channel. Moreover we demonstrate in section 5.3 that these bound states are always tachyonic (i.e. have negative squared mass). As a consequence, such expansion points are maxima in the potential of the field that creates these bound states (in this case $\bar{\phi} \phi$ ), explaining the instability of the corresponding solutions of the gap equation.

It turns out that our effective potential is unbounded from below in the limit $(\bar{\phi} \phi)_{\mathrm{cl}} \rightarrow$ $+\infty$ when $x_{6}<\phi_{1}$. Here $x_{6}$ is the parameter that governs the $\phi^{6}$ interaction of the theory defined precisely in (2.1), and $\phi_{1}$ is a particular function of the 't Hooft coupling $\lambda_{B}$ of this theory listed in (5.11). When $(\bar{\phi} \phi)_{\mathrm{cl}} \rightarrow-\infty$, on the other hand, the potential turns out to be unbounded from below when $x_{6}>\phi_{2} ; \phi_{2}$ is given in (5.11). It follows that the RB theory is unstable - i.e. does not have a stable vacuum state - if either of the conditions above are met. Happily it turns out that $\phi_{1}<\phi_{2}$ so that there is a range of values for $x_{6}$, namely

$$
\phi_{1} \leq x_{6} \leq \phi_{2},
$$

over which the regular boson theory is stable.

The zero temperature phase diagram of the RB theory was worked out in great detail in the recent paper [3]. In order to accomplish this, the authors of [3] evaluated every solution of the gap equation of the RB theory and then compared their free energies. The 
dominant phase at any given values of microscopic parameters is simply the solution with the lowest free energy; this dominant solution was determined in [3] by performing detailed computations. In section 5.4 of this paper we demonstrate that the structure of the phase diagrams presented in [3] has a simple intuitive explanation in terms of the exact LandauGinzburg effective potential for $(\bar{\phi} \phi)_{\mathrm{cl}}$ described above. As we explain in section 5.4 below, the general structure of the phase diagram follows from qualitative curve plotting considerations and can be deduced without performing any detailed computations. Moreover the analysis of the current paper has an added advantage; it allows us to distinguish regions of the phase diagram where the dominant phase is merely metastable (this happens when $x_{6}>\phi_{2}$ or $\left.x_{6}<\phi_{1}\right)$ from regions in the phase diagram in which the dominant solution of the gap equation is truly stable (this happens in the range (1.1)).

\section{Review of known results}

\subsection{Theories and the conjectured duality map}

The RB theory is defined by the action

$$
\begin{aligned}
S_{B}= & \int d^{3} x\left[i \varepsilon^{\mu \nu \rho} \frac{\kappa_{B}}{4 \pi} \operatorname{Tr}\left(X_{\mu} \partial_{\nu} X_{\rho}-\frac{2 i}{3} X_{\mu} X_{\nu} X_{\rho}\right)+D_{\mu} \bar{\phi} D^{\mu} \phi\right. \\
& \left.+m_{B}^{2} \bar{\phi} \phi+\frac{4 \pi b_{4}}{\kappa_{B}}(\bar{\phi} \phi)^{2}+\frac{(2 \pi)^{2}}{\kappa_{B}^{2}}\left(x_{6}^{B}+1\right)(\bar{\phi} \phi)^{3}\right]
\end{aligned}
$$

while the $\zeta_{F}$ and $\zeta_{F}^{2}$ deformed critical fermion $(\mathrm{CF})$ theory is defined by the Lagrangian

$$
\begin{aligned}
S_{F}= & \int d^{3} x\left[i \varepsilon^{\mu \nu \rho} \frac{\kappa_{F}}{4 \pi} \operatorname{Tr}\left(X_{\mu} \partial_{\nu} X_{\rho}-\frac{2 i}{3} X_{\mu} X_{\nu} X_{\rho}\right)+\bar{\psi} \gamma_{\mu} D^{\mu} \psi\right. \\
& \left.-\frac{4 \pi}{\kappa_{F}} \zeta_{F}\left(\bar{\psi} \psi-\frac{\kappa_{F} y_{2}^{2}}{4 \pi}\right)-\frac{4 \pi y_{4}}{\kappa_{F}} \zeta_{F}^{2}+\frac{(2 \pi)^{2}}{\kappa_{F}^{2}} x_{6}^{F} \zeta_{F}^{3}\right] .
\end{aligned}
$$

In these formulae

$$
\kappa_{B}=\operatorname{sgn}\left(k_{B}\right)\left(\left|k_{B}\right|+N_{B}\right), \quad \kappa_{F}=\operatorname{sgn}\left(k_{F}\right)\left(\left|k_{F}\right|+N_{F}\right) .
$$

The levels $k_{F}$ and $k_{B}$ are defined to be the levels of the WZW theory dual to the pure Chern-Simons theory (throughout this paper we work with the dimensional regularisation scheme). For concreteness, in this paper we will assume that the bosonic theory gauge group is $\mathrm{SU}\left(N_{B}\right)$ while the fermionic gauge group is $\mathrm{U}\left(N_{F}\right)$ with 'equal' levels $k_{F}$ for the $\mathrm{SU}\left(N_{F}\right)$ and $\mathrm{U}(1)$ parts of the gauge group. The generalisation to $\mathrm{U}\left(N_{B}\right) \leftrightarrow \mathrm{SU}\left(N_{F}\right)$ and $\mathrm{U}\left(N_{B}\right) \leftrightarrow \mathrm{U}\left(N_{F}\right)$ dualities is straightforward at large $N$ and will not be explicitly considered in this paper.

In the rest of this paper we will present our formulae in terms of the 't Hooft couplings defined by

$$
\lambda_{B}=\frac{N_{B}}{\kappa_{B}}, \quad \lambda_{F}=\frac{N_{F}}{\kappa_{F}} .
$$


We have already mentioned in the introduction that the two theories above have been conjectured to be dual to each other under the level-rank duality map

$$
N_{B}=\left|\kappa_{F}\right|-N_{F}, \quad \kappa_{B}=-\kappa_{F} .
$$

This implies that the bosonic 't Hooft coupling is given in terms of its fermionic counterpart by

$$
\lambda_{B}=\lambda_{F}-\operatorname{sgn}\left(\lambda_{F}\right),
$$

The relations (2.5) and (2.6) are expected to hold even at finite $N$. On the other hand the map between deformations of these two theories is conjectured to be ${ }^{6}$

$$
x_{6}^{F}=x_{6}^{B}, \quad y_{4}=b_{4}, \quad y_{2}^{2}=m_{B}^{2} .
$$

The above equation (2.7) is known to hold only in the large $N$ limit; this relationship may well receive corrections in a power series expansion in $\frac{1}{N}$.

To end this subsection, let us note that under the field redefinition

$$
\phi=\sqrt{\kappa_{B}} \varphi
$$

the action (2.1) turns into

$$
\begin{aligned}
S_{B}= & \frac{N_{B}}{\lambda_{B}} \int d^{3} x\left[i \varepsilon^{\mu \nu \rho} \frac{1}{4 \pi} \operatorname{Tr}\left(X_{\mu} \partial_{\nu} X_{\rho}-\frac{2 i}{3} X_{\mu} X_{\nu} X_{\rho}\right)+D_{\mu} \bar{\varphi} D^{\mu} \varphi\right. \\
& \left.+m_{B}^{2} \bar{\varphi} \varphi+4 \pi b_{4}(\bar{\varphi} \varphi)^{2}+(2 \pi)^{2}\left(x_{6}^{B}+1\right)(\bar{\varphi} \varphi)^{3}\right] .
\end{aligned}
$$

It follows immediately that in the limit

$$
\lambda_{B} \rightarrow 0, \quad m_{B}^{2}, b_{4}, x_{6}=\text { fixed }
$$

the theory (2.1) should reduce to a nonlinear but classical theory of the fields $\varphi$ and $X_{\mu}$. We will return to this point below.

\subsection{Structure of the thermal partition function}

As explained in e.g. [1], the large $N$ thermal free energy of either of these theories on $S^{2} \times S^{1}$ can be obtained following a two step process. In the first step we compute the free energy of the theory in question on $\mathbb{R}^{2} \times S^{1}$, at a fixed value of the gauge holonomies around $S^{1}$. The result is a functional of the holonomy eigenvalue distribution function $\rho(\alpha)$ and is given by the schematic equation

$$
e^{-\mathcal{V}_{2} T^{2} v[\rho]}=\int_{\mathbb{R}^{2} \times S^{1}}[d \phi] e^{-S[\phi, \rho]} .
$$

where $\mathcal{V}_{2}$ is the volume of two dimensional space and $T$ is the temperature.

\footnotetext{
${ }^{6}$ Since $x_{6}^{B}=x_{6}^{F}$, we drop the superscript ${ }^{B}$ or ${ }^{F}$ on $x_{6}$ often in the paper when referring to this coupling.
} 
In order to complete the evaluation of the $S^{2} \times S^{1}$ partition function of interest, in the next step we are instructed to evaluate the unitary matrix integral

$$
\mathcal{Z}_{S^{2} \times S^{1}}=\int[d U]_{\mathrm{CS}} e^{-\mathcal{V}_{2} T^{2} v[\rho]} .
$$

where $[d U]_{\mathrm{CS}}$ is the Chern-Simons modified Haar measure over $\mathrm{U}(N)$ described in [7].

It was demonstrated in [7] that the thermal partition functions (2.12) of the bosonic and fermionic theories agree with each other in the large $N$ limit provided that under duality

$$
v_{F}\left[\rho_{F}\right]=v_{B}\left[\rho_{B}\right],
$$

where the bosonic and fermionic eigenvalue distribution functions, $\rho_{B}$ and $\rho_{F}$, are related via

$$
\left|\lambda_{B}\right| \rho_{B}(\alpha)+\left|\lambda_{F}\right| \rho_{F}(\pi-\alpha)=\frac{1}{2 \pi} .
$$

In this paper we will evaluate the free energy $v_{B}\left[\rho_{B}\right]$ of the bosonic theory in the Higgsed phase and verify (2.13), thus establishing the equality of thermal free energies of the $\mathrm{RB}$ and $\mathrm{CF}$ theories in the Higgsed phase. We summarise the map between the parameters (2.5), (2.6), (2.7) and the holonomy distributions (2.14):

$$
\begin{aligned}
& N_{F}=\left|\kappa_{B}\right|-N_{B}, \quad \kappa_{F}=-\kappa_{B}, \quad \lambda_{F}=\lambda_{B}-\operatorname{sgn}\left(\lambda_{B}\right), \\
& x_{6}^{F}=x_{6}^{B}, \quad y_{4}=b_{4}, \quad y_{2}^{2}=m_{B}^{2}, \quad\left|\lambda_{B}\right| \rho_{B}(\alpha)+\left|\lambda_{F}\right| \rho_{F}(\pi-\alpha)=\frac{1}{2 \pi} \text {. }
\end{aligned}
$$

In appendix A we provide a comprehensive review of everything that is known about the large $N$ thermal free energies of the $\mathrm{CF}$ and $\mathrm{RB}$ theories (the appendix also contains a formula for a 'three variable off-shell' free energy functional of the CF theory that is valid in both phases (A.3)). In the rest of this section we only present those results that will be of relevance for the computations in the paper.

The free energy $v_{F}\left[\rho_{F}\right]$ in the critical fermion theory has been computed in both fermionic phases in [2]. The result is given in terms of an auxiliary off-shell free energy ${ }^{7}$ (equation (A.12) in appendix A)

$$
\begin{aligned}
F_{F}\left(c_{F}, \zeta_{F}\right)= & \frac{N_{F}}{6 \pi}\left[\frac{\left|\lambda_{F}\right|-\operatorname{sgn}\left(\lambda_{F}\right) \operatorname{sgn}\left(X_{F}\right)}{\left|\lambda_{F}\right|} \hat{c}_{F}^{3}-\frac{3}{2 \lambda_{F}}\left(\frac{4 \pi}{\kappa_{F}} \hat{\zeta}_{F}\right) \hat{c}_{F}^{2}\right. \\
& +\frac{1}{2 \lambda_{F}}\left(\frac{4 \pi}{\kappa_{F}} \hat{\zeta}_{F}\right)^{3}+\frac{6 \pi \hat{y}_{2}^{2}}{\kappa_{F} \lambda_{F}} \hat{\zeta}_{F}-\frac{24 \pi^{2} \hat{y}_{4}}{\kappa_{F}^{2} \lambda_{F}} \hat{\zeta}_{F}^{2}+\frac{24 \pi^{3} x_{6}^{F}}{\kappa_{F}^{3} \lambda_{F}} \hat{\zeta}_{F}^{3} \\
& \left.-3 \int_{-\pi}^{\pi} d \alpha \rho_{F}(\alpha) \int_{\hat{c}_{F}}^{\infty} d y y\left(\log \left(1+e^{-y-i \alpha}\right)+\log \left(1+e^{-y+i \alpha}\right)\right)\right] .
\end{aligned}
$$

The above free energy is a function of two variables $c_{F}$ and $\zeta_{F}$. Extremizing $F_{F}$ with respect to these variables and plugging back in the extremum values gives us the free energy $v_{F}\left[\rho_{F}\right]$. The physical interpretation of the variable $c_{F}$ is that its value at the extremum of $F_{F}$ coincides with the pole mass of the fermion.

\footnotetext{
${ }^{7}$ We put a hat over a particular quantity $\left(\right.$ e.g. $\hat{c}_{F}$ ) to denote the dimensionless version of that quantity (e.g. $c_{F}$ ) obtained by multiplying by appropriate powers of the temperature $T$.
} 
The free energy (2.16) assumes two different analytic expressions depending on the $\operatorname{sign} \operatorname{sgn}\left(\lambda_{F}\right) \operatorname{sgn}\left(X_{F}\right)$ and governs the dynamics of the two different phases. The phase in which $\operatorname{sgn}\left(X_{F}\right) \operatorname{sgn}\left(\lambda_{F}\right)= \pm 1$ is referred to as the unHiggsed phase and the Higgsed phase respectively. In equation (A.3) in appendix A, we give an off-shell free energy in terms of three variables (which include $c_{F}$ and $\zeta_{F}$ ) which is analytic in all three variables and encompasses the behaviour of both phases.

The free energy (2.16) in the unHiggsed phase of the CF theory matches the free energy of the regular boson theory in the unHiggsed phase (equation (A.13) in appendix A) computed in [2] under the duality map (2.15). The free energy (2.16) with $\operatorname{sgn}\left(\lambda_{F}\right) \operatorname{sgn}\left(X_{F}\right)=$ -1 gives a prediction for the regular boson theory in the Higgsed phase. Applying the duality transformation (2.15) and making the following 'field' redefinitions:

$$
c_{F}=c_{B}, \quad \frac{4 \pi \zeta_{F}}{\kappa_{F}}=-2 \lambda_{B} \sigma_{B},
$$

we get the following prediction for the free energy in the Higgsed phase (equation (A.28)):

$$
\begin{aligned}
F_{B}\left(c_{B}, \sigma_{B}\right)= & \frac{N_{B}}{6 \pi}\left[-\frac{\lambda_{B}-2 \operatorname{sgn}\left(\lambda_{B}\right)}{\lambda_{B}} \hat{c}_{B}^{3}-3 \hat{\sigma}_{B}\left(\hat{c}_{B}^{2}-\hat{m}_{B}^{2}\right)+6 \hat{b}_{4} \lambda_{B} \hat{\sigma}_{B}^{2}+\left(3 x_{6}^{B}+4\right) \lambda_{B}^{2} \hat{\sigma}_{B}^{3}\right. \\
& \left.+3 \int_{-\pi}^{\pi} d \alpha \rho_{B}(\alpha) \int_{\hat{c}_{B}}^{\infty} d y y\left(\log \left(1-e^{-y-i \alpha}\right)+\log \left(1-e^{-y+i \alpha}\right)\right)\right] .
\end{aligned}
$$

The extremum value of the variable $c_{B}$ corresponds to the pole mass of the $\mathrm{W}$ boson excitation in the Higgsed phase. In the next section we will independently compute the off-shell free energy of the RB theory, and demonstrate that our answer agrees with (2.18) once we identify the field $\sigma_{B}$ with

$$
\sigma_{B}=2 \pi \frac{\bar{\phi} \phi}{N_{B}}
$$

where $\bar{\phi}$ and $\phi$ respectively stand for the saddle point values of the corresponding fields denoted by the same letters (recall these fields have nonzero saddle point values in the Higgsed phase).

Note. Here and in the rest of the paper, we define the quantities $c_{F}$ and $c_{B}$ to be always positive. In other words, $c_{F, B}$ is shorthand for $\left|c_{F, B}\right|$. This is the same convention used in [2].

\section{The Higgsed phase of the regular boson theory}

\subsection{Lagrangian in unitary gauge}

Consider the following action for the $\mathrm{SU}\left(N_{B}\right)$ regular boson theory:

$$
\begin{aligned}
S_{\mathrm{E}}= & \int d^{3} x\left[i \epsilon^{\mu \nu \rho} \frac{\kappa_{B}}{4 \pi} \operatorname{Tr}\left(X_{\mu} \partial_{\nu} X_{\rho}-\frac{2 i}{3} X_{\mu} X_{\nu} X_{\rho}\right)+D_{\mu} \bar{\phi} D^{\mu} \phi\right. \\
& \left.+m_{B}^{2} \bar{\phi} \phi+\frac{4 \pi b_{4}}{\kappa_{B}}(\bar{\phi} \phi)^{2}+\frac{(2 \pi)^{2}}{\kappa_{B}^{2}}\left(x_{6}^{B}+1\right)(\bar{\phi} \phi)^{3}\right],
\end{aligned}
$$


with $D_{\mu}=\partial_{\mu}-i X_{\mu}$. The above action can be reorganised as follows in the Higgsed phase where we anticipate $\langle\bar{\phi} \phi\rangle \neq 0$. Following [1] we work in the unitary gauge

$$
\phi^{i}(x)=\delta^{i N_{B}} \sqrt{\left|\kappa_{B}\right|} V(x) .
$$

For future reference we note also that (3.2) implies the following for the 'classical' field $\varphi$ defined in (2.8):

$$
\varphi^{i}(x)=\delta^{i N_{B}} \sqrt{\operatorname{sgn}\left(\kappa_{B}\right)} V(x) .
$$

The field $V(x)$ shall be termed the Higgs field. The above gauge choice lets us decompose the gauge field $X_{\mu}$ as

$$
X_{\mu}=\left(\begin{array}{cc}
\left(A_{\mu}\right)^{a}{ }_{b}-\frac{\delta^{a} b}{N_{B}-1} Z_{\mu} & \frac{1}{\sqrt{\kappa_{B}}}\left(W_{\mu}\right)^{a} \\
\frac{1}{\sqrt{\kappa_{B}}}\left(\bar{W}_{\mu}\right)_{b} & Z_{\mu}
\end{array}\right),
$$

where the indices $a, b$ run over $1, \ldots, N_{B}-1$. In terms of these variables, the action can be rewritten as follows: ${ }^{8}$

$$
\begin{aligned}
S_{\mathrm{E}}[A, W, Z, V]= & \frac{i \kappa_{B}}{4 \pi} \int \operatorname{Tr}\left(A d A-\frac{2 i}{3} A A A\right) \\
& +\frac{i}{4 \pi} \int\left(2 \bar{W}_{a} d W^{a}+\kappa_{B} Z d Z-2 i Z \bar{W}_{a} W^{a}-2 i \bar{W}_{a} A^{a}{ }_{b} W^{b}\right) \\
& +\int d^{3} x\left(\left|\kappa_{B}\right| V^{2} Z_{\mu} Z^{\mu}+\operatorname{sgn}\left(\kappa_{B}\right) V^{2} \bar{W}_{a \mu} W^{a \mu}\right) \\
& +\left|\kappa_{B}\right| \int d^{3} x\left(\partial_{\mu} V \partial^{\mu} V+m_{B}^{2} V^{2}+4 \pi b_{4} \operatorname{sgn}\left(\kappa_{B}\right) V^{4}+4 \pi^{2}\left(x_{6}^{B}+1\right) V^{6}\right) .
\end{aligned}
$$

\subsection{An effective action for the Higgs field $V$}

We will now compute the thermal partition function of the regular boson theory in the Higgsed phase, i.e. we will compute $v_{B}\left[\rho_{B}\right]$ defined by

$$
e^{-\mathcal{V}_{2} T^{2} v_{B}\left[\rho_{B}\right]}=\int_{\mathbb{R}^{2} \times S^{1}}[d V d W d Z d A] e^{-S_{\mathrm{E}}[A, W, Z, V]},
$$

where $S_{\mathrm{E}}[A, W, Z, V]$ was defined in (3.5). For this purpose it is convenient to break up the effective action $S_{\mathrm{E}}[A, W, Z, V]$ into two parts

$$
S_{\mathrm{E}}[A, W, Z, V]=S_{1}[A, W, Z, V]+S_{2}[V]
$$

where

$$
\begin{aligned}
S_{1}[A, W, Z, V]= & \frac{i \kappa_{B}}{4 \pi} \int d^{3} x \operatorname{Tr}\left(A d A-\frac{2 i}{3} A A A\right) \\
& +\frac{i}{4 \pi} \int\left(2 \bar{W}_{a} d W^{a}+\kappa_{B} Z d Z-2 i Z \bar{W}_{a} W^{a}-2 i \bar{W}_{a} A^{a}{ }_{b} W^{b}\right) \\
& +\int d^{3} x\left(\left|\kappa_{B}\right| V^{2} Z_{\mu} Z^{\mu}+\operatorname{sgn}\left(\kappa_{B}\right) V^{2} \bar{W}_{a}^{\mu} W_{\mu}^{a}\right),
\end{aligned}
$$

\footnotetext{
${ }^{8}$ The notation $A B C$ stands for $d^{3} x \epsilon^{\mu \nu \rho} A_{\mu} B_{\nu} C_{\rho}$.
} 
and

$$
\begin{aligned}
S_{2}[V] & =\int d^{3} x\left(\left|\kappa_{B}\right| \partial_{\mu} V \partial^{\mu} V+U_{\mathrm{cl}}(V)\right), \\
U_{\mathrm{cl}}(V) & =\left|\kappa_{B}\right| m_{B}^{2} V^{2}+4 \pi b_{4} \kappa_{B} V^{4}+4 \pi^{2}\left|\kappa_{B}\right|\left(x_{6}^{B}+1\right) V^{6} .
\end{aligned}
$$

The path integral (2.11) can be rewritten as

$$
e^{-\mathcal{V}_{2} T^{2} v_{B}\left[\rho_{B}\right]}=\int[d V] e^{-S_{2}[V]} \int[d W d Z d A] e^{-S_{1}[A, W, Z, V]}
$$

Let us first study 'inner' path integral i.e.

$$
e^{-\mathcal{V}_{2} T^{2} v_{\mathrm{CB}}\left[\rho_{B}, V\right]} \equiv \int[d W d Z d A] e^{-S_{1}[A, W, Z, V]},
$$

where the right hand side defines the quantity $v_{\mathrm{CB}}\left[\rho_{B}, V\right]$. As far as the path integral in (3.11) is concerned, $V(x)$ is a background field. The path integral (3.11) is difficult to evaluate for arbitrary $V(x)$ even in the large $N_{B}$ limit. ${ }^{9}$ This problem simplifies, however, in the special case that $V(x)$ is a constant. In fact, precisely in this limit, the path integral (3.11) has been evaluated in the recent paper [1]. Luckily, it will turn out that, in the large $N$ limit, the integral over $V(x)$ in (3.10) localises to a saddle point at which $V(x)$ is constant (see below). As a consequence we only need the result of the path integral (3.11) for constant $V(x)$; we are able to read off this result directly from [1] which we now pause to recall.

The authors of [1] studied the critical boson theory in its Higgsed phase. Working in unitary gauge and following manipulations essentially identical to those outlined in the previous subsection, they found that the CB theory in the Higgsed phase can be rewritten as effective theory of interacting massive $W$ bosons, $Z$ bosons and $\mathrm{SU}\left(N_{B}-1\right)$ gauge fields, whose action is given by

$$
\begin{aligned}
S_{\mathrm{E}}[A, W, Z]= & \frac{i \kappa_{B}}{4 \pi} \int \operatorname{Tr}\left(A d A-\frac{2 i}{3} A A A\right) \\
& +\frac{i}{4 \pi} \int\left(2 \bar{W}_{a} d W^{a}+\kappa_{B} Z d Z-2 i Z \bar{W}_{a} W^{a}-2 i \bar{W}_{a} A_{b}^{a} W^{b}\right) \\
& -\int d^{3} x\left(\frac{N_{B}}{4 \pi} m_{B}^{\mathrm{cri}} Z_{\mu} Z^{\mu}+\frac{\lambda_{B}}{4 \pi} m_{B}^{\mathrm{cri}} \bar{W}_{a \mu} W^{a \mu}\right) .
\end{aligned}
$$

The authors of [1] were then able to evaluate the finite temperature partition function for the theory defined by (3.12). Their final result for $v_{\mathrm{CB}}\left[\rho_{B}\right]$ is given as follows. One obtains $v_{\mathrm{CB}}\left[\rho_{B}\right]$ by extremizing an off-shell free energy $F_{\mathrm{CB}}\left(c_{B}\right)$ given by

$$
\begin{aligned}
F_{\mathrm{CB}}\left(c_{B}\right)= & \frac{N_{B}}{6 \pi}\left[-\frac{\lambda_{B}-2 \operatorname{sgn}\left(\lambda_{B}\right)}{\lambda_{B}} \hat{c}_{B}^{3}+\frac{3}{2} \hat{m}_{B}^{\mathrm{cri}} \hat{c}_{B}^{2}+\Lambda\left(\hat{m}_{B}^{\mathrm{cri}}\right)^{3}\right. \\
& \left.+3 \int_{-\pi}^{\pi} d \alpha \rho_{B}(\alpha) \int_{\hat{c}_{B}}^{\infty} d y y\left(\log \left(1-e^{-y-i \alpha}\right)+\log \left(1-e^{-y+i \alpha}\right)\right)\right],
\end{aligned}
$$

\footnotetext{
${ }^{9} \mathrm{Eq}$. (3.11) is effectively the generating function of all correlation functions of the dimension two scalar $J_{0}$ in the large $N$ critical boson theory, and so contains a great deal of information.
} 
Here, $\Lambda$ is an undetermined constant; shifts in $\Lambda$ correspond to shifts in the cosmological constant counterterm in the starting action for the CB theory (see [1] for a discussion).

Note that the action $S_{1}$ in (3.5) agrees precisely with the action (3.12) reported in [1] if we replace $m_{B}^{\text {cri }}$ by the quantity

$$
m_{B}^{\text {cri }}=-\frac{4 \pi}{\left|\lambda_{B}\right|} V^{2} \quad \text { with } V \text { constant. }
$$

It follows that for the special case that $V(x)$ is constant, the path integral (3.11) is given by the extremum value of (3.13) with the replacement (3.14) and the path integral over $V$ in (3.10) takes the form

$$
\int[d V] e^{-S_{\mathrm{eff}}[V]} \quad \text { with } \quad S_{\mathrm{eff}}[V]=S_{2}[V]+\mathcal{V}_{2} T^{2} v_{\mathrm{CB}}\left[\rho_{B}, V\right]
$$

From the expressions for $S_{2}[V],{ }^{10}$ and $F_{B}\left(c_{B}\right)$ in (3.9) and (3.13), it is clear that there is an overall factor of $N_{B}$ in front of the effective action $S_{\text {eff }}[V]$. In the large $N_{B}$ limit the path integral over $V$ may be evaluated in the saddle-point approximation. We expect the dominant minima of the effective action to occur at constant values of $V$ since the kinetic term $\partial_{\mu} V \partial^{\mu} V$ adds a positive definite piece to the action. For this reason it is sufficient to have the expression $v_{\mathrm{CB}}\left[\rho_{B}, V\right]$ only at constant $V$. As we have already explained above, this result is given by extremizing (3.13) w.r.t. $c_{B}$ after making the replacement (3.14). It follows that the final result for $v_{B}\left[\rho_{B}\right]$ in (3.6) is obtained by extremizing the regular boson off-shell free energy

$$
F_{B}\left(c_{B}, V\right)=F_{\mathrm{CB}}\left(c_{B}\right)+\frac{1}{\mathcal{V}_{2} T^{2}} S_{2}[V]
$$

with respect to both $c_{B}$ and $V .{ }^{11}$ Using the explicit expressions (3.9), (3.13) and (3.14) we find the following explicit result for the off-shell free energy of the RB theory:

$$
\begin{aligned}
F_{B}\left(c_{B}, V\right)= & \frac{N_{B}}{6 \pi}\left[-\frac{\left(\lambda_{B}-2 \operatorname{sgn}\left(\lambda_{B}\right)\right)}{\lambda_{B}} \hat{c}_{B}^{3}-\frac{8 \Lambda}{\left|\lambda_{B}\right|^{3}}\left(2 \pi \hat{V}^{2}\right)^{3}\right. \\
& -\frac{3}{\left|\lambda_{B}\right|}\left(\hat{c}_{B}^{2}-\hat{m}_{B}^{2}\right) 2 \pi \hat{V}^{2}+\frac{6 \hat{b}_{4}}{\lambda_{B}}\left(2 \pi \hat{V}^{2}\right)^{2}+\frac{\left(3 x_{6}^{B}+3\right)}{\left|\lambda_{B}\right|}\left(2 \pi \hat{V}^{2}\right)^{3} \\
& \left.+3 \int_{-\pi}^{\pi} \rho_{B}(\alpha) d \alpha \int_{\hat{c}_{B}}^{\infty} d y y\left(\log \left(1-e^{-y-i \alpha}\right)+\log \left(1-e^{-y+i \alpha}\right)\right)\right] .
\end{aligned}
$$

We compare the result (3.16) with the prediction (2.18) of duality for the Higgsed phase

\footnotetext{
${ }^{10}$ In [1], the free energy $v_{\mathrm{CB}}\left[\rho_{B}\right]$ depended on $m_{B}^{\text {cri }}$ which was a parameter in the theory. After the replacement (3.14), the dependence on the parameter $m_{B}^{\text {cri }}$ is replaced by a dependence on the field $V(x)$. We have included an explicit $V$ in the notation for $v_{\mathrm{CB}}\left[\rho_{B}, V\right]$ to highlight this dependence on the Higgs field $V$.

${ }^{11}$ It is understood that $F_{\mathrm{CB}}\left[\rho_{B}, c_{B}\right]$ is evaluated after making the replacement (3.14).
} 
free energy given by

$$
\begin{aligned}
F_{B}\left(c_{B}, V\right)= & \frac{N_{B}}{6 \pi}\left[-\frac{\lambda_{B}-2 \operatorname{sgn}\left(\lambda_{B}\right)}{\lambda_{B}} \hat{c}_{B}^{3}\right. \\
& -\frac{3}{\left|\lambda_{B}\right|}\left(\hat{c}_{B}^{2}-\hat{m}_{B}^{2}\right) 2 \pi \hat{V}^{2}+\frac{6 \hat{b}_{4}}{\lambda_{B}}\left(2 \pi \hat{V}^{2}\right)^{2}+\frac{\left(3 x_{6}^{B}+4\right)}{\left|\lambda_{B}\right|}\left(2 \pi \hat{V}^{2}\right)^{3} \\
& \left.+3 \int_{-\pi}^{\pi} d \alpha \rho_{B}(\alpha) \int_{\hat{c}_{B}}^{\infty} d y y\left(\log \left(1-e^{-y-i \alpha}\right)+\log \left(1-e^{-y+i \alpha}\right)\right)\right],
\end{aligned}
$$

where, to get the above expression, we have used (3.2) and (2.19) to write the field $\sigma_{B}$ in $(2.18)$ as

$$
\sigma_{B}=\frac{2 \pi V^{2}}{\left|\lambda_{B}\right|} .
$$

We see that (3.16) agrees precisely with (3.17) provided we choose the as yet undetermined parameter $\Lambda$ as

$$
\Lambda=-\frac{1}{8} \lambda_{B}^{2}
$$

In the next subsection we will verify that the result (3.19) - which is so far just a prediction of duality - can also be obtained by direct computation within the Higgsed CB theory. The strategy we employ is the following. We first note that the gap equation corresponding to stationarity of (3.16) with respect to $\hat{V}^{2}$ is

$$
\hat{c}_{B}^{2}-\hat{m}_{B}^{2}+8 \Lambda \hat{\sigma}_{B}^{2}-4 \hat{b}_{4} \lambda_{B} \hat{\sigma}_{B}-\left(3 x_{6}^{B}+3\right) \lambda_{B}^{2} \hat{\sigma}_{B}^{2}=0 .
$$

where $\sigma_{B}$ is given in terms of $V^{2}$ by (3.18). The equation (3.20) merely simply expresses the condition that the tadpole of the fluctuation of the scalar field $V$ vanishes when the field $V$ is expanded around its true solution $v$. In the next subsection we directly evaluate this 'tadpole vanishing condition' in the RB theory in the Higgsed phase and thereby determine $\Lambda$ by comparison with (3.20).

\subsection{Tadpole cancellation for $V$}

As we have explained above, in the Higgsed phase our scalar field $V$ gets the expectation value $v$. It is useful to define

$$
V(x)=v+H(x) .
$$

The condition that $v$ is the correct vacuum expectation value of $V(x)$ is equivalent to the condition that the expectation value (i.e. one point function, i.e. tadpole) of the fluctuation $H(x)$ vanishes. In other words we require that

$$
\int_{\mathbb{R}^{2} \times S^{1}}[d V d W d Z d A] H(x) e^{-S_{\mathrm{E}}[A, W, Z, V]}=0 .
$$

Using the explicit form of $S_{\mathrm{E}}[A, W, Z, V]$ in (3.5), equation (3.22) can be rewritten as

$$
\operatorname{sgn}\left(\kappa_{B}\right)\left\langle\bar{W}_{a \mu}(x) W^{a \mu}(x)\right\rangle+\left|\kappa_{B}\right|\left\langle Z_{\mu}(x) Z^{\mu}(x)\right\rangle+\frac{\partial}{\partial\left(v^{2}\right)} U_{\mathrm{cl}}\left(v^{2}\right)=0 .
$$


where $U_{\mathrm{cl}}(V)$ is the potential for the Higgs field $V$ given in (3.9) and all expectation values are evaluated about the 'vacuum' where $V(x)=v$. While the first and third terms in (3.23) above are both of order $N_{B}$, it is easily verified that the second term in this equation the term proportional to $\left\langle Z_{\mu}(x)^{2}\right\rangle$ is of order unity ${ }^{12}$ and so can be dropped in the large $N_{B}$ limit. At leading order in the large $N_{B}$ limit, it follows that the tadpole cancellation condition (3.22) can be rewritten as

$$
\frac{\lambda_{B}}{2 \pi \mathcal{V}_{3}} \int d^{3} x\left\langle\bar{W}_{a \mu}(x) W^{a \mu}(x)\right\rangle+\frac{\partial U_{\mathrm{cl}}\left(\sigma_{B}\right)}{\partial \sigma_{B}}=0,
$$

where we have integrated the equation (3.23) over spacetime and have divided the resulting expression by the volume of spacetime $\mathcal{V}_{3}$. We have also changed variables to $\sigma_{B}=2 \pi v^{2} /\left|\lambda_{B}\right|$ defined in (3.18). Moving to momentum space, (3.24) turns into

$$
\frac{\lambda_{B}}{2 \pi} \int \frac{\mathcal{D}^{3} p}{(2 \pi)^{3}} \eta^{\mu \nu} G_{a \mu \nu}^{a}(p)+\frac{\partial U_{\mathrm{cl}}\left(\sigma_{B}\right)}{\partial \sigma_{B}}=0,
$$

where

$$
\left\langle\bar{W}_{a \mu}(-p) W_{\nu}^{b}\left(p^{\prime}\right)\right\rangle=G_{a \mu \nu}^{b}(p)(2 \pi)^{3} \delta^{(3)}\left(p-p^{\prime}\right)=\delta_{a}^{b} G_{\mu \nu}(p)(2 \pi)^{3} \delta^{(3)}\left(p-p^{\prime}\right),
$$

and the measure $\mathcal{D}$ is the natural measure in momentum space at finite temperature. ${ }^{13}$ Happily, the exact all-orders formula for the propagator $G_{\mu \nu}$ was computed in [1]. In appendix B we proceed to plug the explicit expression for $G_{\mu \nu}$ and evaluate the first term in (3.25). We are able to evaluate all the relevant summations and integrals analytically, and demonstrate that in our choice of regularisation scheme (3.24) takes the explicit form

$$
-\frac{N_{B}}{2 \pi}\left(c_{B}^{2}-\lambda_{B}^{2} \sigma_{B}^{2}\right)+\frac{\partial U_{\mathrm{cl}}\left(\sigma_{B}\right)}{\partial \sigma_{B}}=0 .
$$

Recall the expression for $\mathrm{U}\left(\sigma_{B}\right)$ from (3.9):

$$
U_{\mathrm{cl}}\left(\sigma_{B}\right)=\frac{N_{B}}{2 \pi}\left(m_{B}^{2} \sigma_{B}+2 b_{4} \lambda_{B} \sigma_{B}^{2}+\left(x_{6}^{B}+1\right) \lambda_{B}^{2} \sigma_{B}^{3}\right) .
$$

Plugging this back into (3.28) we find

$$
c_{B}^{2}-m_{B}^{2}-4 b_{4} \lambda_{B} \sigma_{B}-\left(3 x_{6}^{B}+4\right) \lambda_{B}^{2} \sigma_{B}^{2}=0,
$$

Comparing this with the gap equation obtained earlier in (3.20), we see that (3.20) matches (3.30) for the predicted value of $\Lambda=-\lambda_{B}^{2} / 8$ in (3.19) as expected.

\footnotetext{
${ }^{12}$ This follows from the observation that the $Z$ propagator scales like $1 / N_{B}$.

${ }^{13}$ Explicitly, the notation $\mathcal{D}^{3} p$ signifies that we work at finite temperature i.e. on the spacetime $\mathbb{R}^{2} \times S_{\beta}^{1}$ where the third direction $x^{3}$ is a circle of circumference $\beta$. The measure $\mathcal{D}^{3} p$ is then given by

$$
\int \frac{\mathcal{D}^{3} p}{(2 \pi)^{3}} f(p)=\int \frac{d p_{1} d p_{2}}{(2 \pi)^{2}} \int_{-\pi}^{\pi} d \alpha \rho_{B}(\alpha) \frac{1}{\beta} \sum_{n=-\infty}^{\infty} f\left(\frac{2 \pi n+\alpha}{\beta}\right)
$$

where $\rho_{B}(\alpha)$ is the distribution of the eigenvalues $\alpha$ of the gauge field holonomy around $S_{\beta}^{1}$. 


\section{A three variable off-shell free energy}

The finite temperature unHiggsed phase is governed by the two-variable off-shell free energy (equation (A.14) in appendix A)

$$
\begin{aligned}
F_{B}\left(c_{B}, \tilde{\mathcal{S}}\right)= & \frac{N_{B}}{6 \pi}\left[-\hat{c}_{B}^{3}+3 \tilde{\mathcal{S}}\left(\hat{c}_{B}^{2}-\hat{m}_{B}^{2}\right)+6 \hat{b}_{4} \lambda_{B} \tilde{\mathcal{S}}^{2}-\left(4+3 x_{6}^{B}\right) \lambda_{B}^{2} \tilde{\mathcal{S}}^{3}\right. \\
& \left.+3 \int_{-\pi}^{\pi} d \alpha \rho_{B}(\alpha) \int_{\hat{c}_{B}}^{\infty} d y y\left(\log \left(1-e^{-y-i \alpha}\right)+\log \left(1-e^{-y+i \alpha}\right)\right)\right],
\end{aligned}
$$

On the other hand, we have demonstrated in this paper that the finite temperature Higgsed phase is governed by the two-variable off-shell free energy (2.18). As these are two separate 'phases' of the same theory it is somewhat unsatisfying that the off-shell 'Landau-Ginzburg' free energies used to describe them are different. The reader may wonder whether there exists a single master off-shell free energy functional — analytic in all 'fields' — which encompasses the physics of both (4.1) and (2.18). At least at the algebraic level there is a simple affirmative answer to this question as we now describe.

Consider the off-shell free energy

$$
\begin{aligned}
F\left(c_{B}, \sigma_{B}, \tilde{\mathcal{S}}\right)= & \frac{N_{B}}{6 \pi}\left[-\hat{c}_{B}^{3}-4 \tilde{\mathcal{S}}^{3} \lambda_{B}^{2}-3 \hat{c}_{B}^{2} \hat{\sigma}_{B}-12 \tilde{\mathcal{S}}^{2} \lambda_{B}^{2} \hat{\sigma}_{B}-12 \tilde{\mathcal{S}} \lambda_{B}^{2} \hat{\sigma}_{B}^{2}\right. \\
& +6 \hat{c}_{B}\left|\lambda_{B}\right|\left(\tilde{\mathcal{S}}+\hat{\sigma}_{B}\right)^{2}+3\left(\hat{m}_{B}^{2} \hat{\sigma}_{B}+2 \lambda_{B} \hat{b}_{4} \hat{\sigma}_{B}^{2}+\lambda_{B}^{2} x_{6}^{B} \hat{\sigma}_{B}^{3}\right) \\
& \left.+3 \int_{-\pi}^{\pi} d \alpha \rho_{B}(\alpha) \int_{\hat{c}_{B}}^{\infty} d y y\left(\log \left(1-e^{-y-i \alpha}\right)+\log \left(1-e^{-y+i \alpha}\right)\right)\right] .
\end{aligned}
$$

Note that (4.2) is a function of three 'field' variables, namely $c_{B}, \tilde{\mathcal{S}}$ and $\sigma_{B}$. Extremizing (4.2) w.r.t. $\tilde{\mathcal{S}}, c_{B}$ and $\sigma_{B}$ respectively yields the equations

$$
\begin{aligned}
\left(\tilde{\mathcal{S}}+\hat{\sigma}_{B}\right)\left(-\hat{c}_{B}+\left|\lambda_{B}\right|\left(\tilde{\mathcal{S}}+\hat{\sigma}_{B}\right)\right) & =0, \\
\hat{c}_{B}\left(\mathcal{S}\left(c_{B}\right)+\hat{\sigma}_{B}\right)-\left|\lambda_{B}\right|\left(\tilde{\mathcal{S}}+\hat{\sigma}_{B}\right)^{2} & =0, \\
\hat{c}_{B}^{2}-\hat{m}_{B}^{2}-4 \hat{c}_{B}\left|\lambda_{B}\right|\left(\tilde{\mathcal{S}}+\hat{\sigma}_{B}\right)+\lambda_{B}\left(4 \tilde{\mathcal{S}}^{2} \lambda_{B}-4 \hat{b}_{4} \hat{\sigma}_{B}+8 \lambda_{B} \hat{\sigma}_{B} \tilde{\mathcal{S}}-3 \lambda_{B} \hat{\sigma}_{B}^{2} x_{6}^{B}\right) & =0 .
\end{aligned}
$$

The quantity $\mathcal{S}\left(c_{B}\right)$ that appears in the second of (4.3) is defined in (A.1). Off-shell, the objects $\mathcal{S}\left(c_{B}\right)$ and $\tilde{\mathcal{S}}$ are completely distinct. $\mathcal{S}\left(c_{B}\right)$ is a function of $c_{B}$ while $\tilde{\mathcal{S}}$ is an independent variable. However it is easy to see (by subtracting the first two equations in (4.3)) that these two quantities are, in fact, equal on-shell.

Note in particular that the first of $(4.3)$ - the equation that follows upon extremizing (4.2) w.r.t. $\tilde{\mathcal{S}}$ - is the product of two factors. This equation is satisfied either if

$$
\tilde{\mathcal{S}}+\hat{\sigma}_{B}=0
$$

or if

$$
-\hat{c}_{B}+\left|\lambda_{B}\right|\left(\tilde{\mathcal{S}}+\hat{\sigma}_{B}\right)=0
$$


(clearly (4.4) and (4.5) cannot simultaneously be obeyed unless $c_{B}=0$ ). Let us first suppose that (4.4) is obeyed. Using (4.4) to eliminate $\sigma_{B}$ from (4.2) yields an off-shell free energy that now depends only on $\tilde{\mathcal{S}}$ and $c_{B}$. It is easily verified that the resultant free energy agrees exactly with the two-variable free energy (4.1) in the unHiggsed phase. It follows that solutions of (4.4) parametrize - and govern the physics of - the unHiggsed phase of the RB theory.

In a similar manner let us now suppose that (4.5) is obeyed in which case we use it to eliminate $\tilde{\mathcal{S}}$. It is easily verified that the resultant two-variable free energy — which depends on $c_{B}$ and $\sigma_{B}$ - agrees exactly with (3.17) with the identification (3.18):

$$
\sigma_{B}=\frac{2 \pi v^{2}}{\left|\lambda_{B}\right|}
$$

It follows that solutions of (4.5) parametrize - and govern the physics of - the Higgsed phase of the RB theory.

The identification (4.6) has a simple explanation. Recall that the bare mass $m_{B}^{2}$ appeared in the action (2.1) as the coefficient of $\bar{\phi} \phi$. It follows that the Legendre transform of the free energy of our theory w.r.t. $m_{B}^{2}$ yields the exact quantum corrected effective potential of our theory as a function of the composite field $(\bar{\phi} \phi)_{\mathrm{cl}}$. This Legendre transform may be computed by adding the term

$$
-m_{B}^{2}(\bar{\phi} \phi)_{\mathrm{cl}}
$$

to (4.2) and then treating $m_{B}^{2}$ as a new dynamical field w.r.t. which (4.2) has to be extremized (of course we also continue to extremize (4.2) w.r.t. $c_{B}, \sigma_{B}$ and $\tilde{\mathcal{S}}$ as before). Note that the dependence of (4.2) on $m_{B}^{2}$ is extremely simple; it occurs entirely through the term $\frac{N_{B}}{2 \pi} \sigma_{B} m_{B}^{2}$. As a consequence, extremizing w.r.t. $m_{B}^{2}$ sets

$$
\sigma_{B}=\frac{2 \pi(\bar{\phi} \phi)_{\mathrm{cl}}}{N_{B}}
$$

In the Higgsed phase it follows from $\phi^{i}=\delta^{i N_{B}} \sqrt{\left|\kappa_{B}\right|} v$ (equation (3.2)) that

$$
(\bar{\phi} \phi)_{\mathrm{cl}}=\left|\kappa_{B}\right| v^{2} \text {. }
$$

(in obtaining (4.8) we use the fact that the Higgs field $V$ is effectively classical in the large $N_{B}$ limit). Inserting (4.8) into (4.7) yields (4.6). We note, however, that (4.7) is more general than (4.6) because it applies even in the unHiggsed phase. We will make use of this fact in the next section.

We have thus found a simple single off-shell free energy — namely (4.2) - that captures the physics of both the Higgsed and the unHiggsed phases. We have also explained that one of the three variables that appears in this free energy - namely $\sigma_{B}$ - has a simple direct physical interpretation given by (4.7). It follows, in particular, that if we integrate $c_{B}$ and $\tilde{\mathcal{S}}$ out from (4.2), the resultant free energy (which is a function of $\sigma_{B}$ ) can be reinterpreted as the quantum effective potential of the theory as a function of $(\bar{\phi} \phi)_{\mathrm{cl}}$. In the next section we will explicitly undertake this exercise in the zero temperature limit. 
It is easily verified that the duality map (2.15) between parameters together with the field redefinitions

$$
\lambda_{B} \tilde{\mathcal{S}}=\lambda_{F} \tilde{\mathcal{C}}-\frac{\operatorname{sgn}\left(\lambda_{F}\right)}{2} \hat{c}_{F}, \quad \lambda_{B} \sigma_{B}=-\frac{2 \pi \zeta_{F}}{\kappa_{F}}, \quad c_{B}=c_{F} .
$$

turns the bosonic off-shell free energy (4.2) into the fermionic off-shell free energy (A.3). This match captures the Bose Fermi duality between RB and CF theories at the level of the complete thermal off-shell free energies of the two theories; note that each of these off-shell free energies is analytic in all 'fields'. In appendix C we investigate the behaviour of our three-variable off-shell free energy in the so called critical boson scaling limit of the RB theory.

\section{The exact Landau-Ginzburg effective potential}

In this section we integrate out the variables $\tilde{\mathcal{S}}$ and $c_{B}$ out from the effective action (4.1) and obtain an off-shell free energy for the field $\sigma_{B}$. We work at zero temperature throughout this section. In this simple - and physically especially important - limit we obtain a simple analytic expression for the resultant free energy as a function of $\sigma_{B}$. As we have explained in the previous section, this free energy is simply related to the quantum effective potential of the RB theory as a function of the field $(\bar{\phi} \phi)_{\mathrm{cl}}$.

After having obtained this exact Landau-Ginzburg potential we study and use it in various ways. First we note that this effective potential has extrema of two sorts - local maxima and local minima. Local maxima represent unstable saddle point solutions of the theory. In the case of the unHiggsed branch (see below) we present an interpretation of the resultant instability in terms of the tachyonic bound states of the system. We also use the exact Landau-Ginzburg effective action that we obtain to understand the zero temperature phase diagram of the RB theory (as a function of its microscopic parameters) in a simple and intuitive way. Finally we also make a prediction for the range of the parameter $x_{6}$ over which the RB theory is stable, i.e. has a stable vacuum.

\subsection{An effective potential for $\sigma_{B}$}

In the zero temperature limit the three-variable off-shell free energy (4.2) simplifies to

$$
\begin{aligned}
F\left(c_{B}, \sigma_{B}, \tilde{\mathcal{S}}\right)= & \frac{N_{B}}{6 \pi}\left[-\hat{c}_{B}^{3}-4 \tilde{\mathcal{S}}^{3} \lambda_{B}^{2}-3 \hat{c}_{B}^{2} \hat{\sigma}_{B}-12 \tilde{\mathcal{S}}^{2} \lambda_{B}^{2} \hat{\sigma}_{B}-12 \tilde{\mathcal{S}} \lambda_{B}^{2} \hat{\sigma}_{B}^{2}\right. \\
& \left.+6 \hat{c}_{B}\left|\lambda_{B}\right|\left(\tilde{\mathcal{S}}+\hat{\sigma}_{B}\right)^{2}+3\left(\hat{m}_{B}^{2} \hat{\sigma}_{B}+2 \lambda_{B} \hat{b}_{4} \hat{\sigma}_{B}^{2}+x_{6}^{B} \lambda_{B}^{2} \hat{\sigma}_{B}^{3}\right)\right] .
\end{aligned}
$$

Varying this free energy w.r.t. $\tilde{\mathcal{S}}$ produces the first of the gap equations in (4.3) which we repeat here for convenience

$$
\left(\tilde{\mathcal{S}}+\hat{\sigma}_{B}\right)\left(-\hat{c}_{B}+\left|\lambda_{B}\right|\left(\tilde{\mathcal{S}}+\hat{\sigma}_{B}\right)\right)=0 .
$$

As we have discussed above, this equation has two solutions corresponding to the unHiggsed and Higgsed branches:

$$
\text { unHiggsed }: \quad \tilde{\mathcal{S}}=-\hat{\sigma}_{B}, \quad \text { Higgsed }: \quad \tilde{\mathcal{S}}=-\hat{\sigma}_{B}+\frac{\hat{c}_{B}}{\left|\lambda_{B}\right|} .
$$


Plugging these solutions back into the expression for the free energy, we have, in the unHiggsed phase,

$$
F^{(\mathrm{uH})}\left(c_{B}, \sigma_{B}\right)=\frac{N_{B}}{6 \pi T^{3}}\left(-c_{B}^{3}+4 \lambda_{B}^{2} \sigma_{B}^{3}-3\left(c_{B}^{2}-m_{B}^{2}\right) \sigma_{B}+6 b_{4} \lambda_{B} \sigma_{B}^{2}+3 x_{6}^{B} \lambda_{B}^{2} \sigma_{B}^{3}\right),
$$

and in the Higgsed phase,

$$
F^{(\mathrm{H})}\left(c_{B}, \sigma_{B}\right)=\frac{N_{B}}{6 \pi T^{3}}\left(\frac{2-\left|\lambda_{B}\right|}{\left|\lambda_{B}\right|} c_{B}^{3}+4 \lambda_{B}^{2} \sigma_{B}^{3}-3\left(c_{B}^{2}-m_{B}^{2}\right) \sigma_{B}+6 b_{4} \lambda_{B} \sigma_{B}^{2}+3 x_{6}^{B} \lambda_{B}^{2} \sigma_{B}^{3}\right) .
$$

We then extremize the above free energies with respect to $c_{B}$ to get

$$
\text { unHiggsed : } \quad c_{B}=-2 \sigma_{B}, \quad \text { Higgsed : } \quad c_{B}=\frac{\left|\lambda_{B}\right|}{2-\left|\lambda_{B}\right|} 2 \sigma_{B} .
$$

Recall that $c_{B}$ is positive by definition. It follows that the solutions (5.6) exist only when $\sigma_{B}$ is positive (negative) in the Higgsed (unHiggsed) phase respectively. Plugging back the above expressions into the free energies in (5.4) and (5.5), we get

$$
F^{(\mathrm{uH})}\left(\sigma_{B}\right)=\frac{N_{B}}{2 \pi T^{3}}\left[\left(\left(1+x_{6}^{B}\right)-\frac{4-\lambda_{B}^{2}}{3 \lambda_{B}^{2}}\right) \lambda_{B}^{2} \sigma_{B}^{3}+2 b_{4} \lambda_{B} \sigma_{B}^{2}+m_{B}^{2} \sigma_{B}\right]
$$

and in the Higgsed phase,

$$
F^{(\mathrm{H})}\left(\sigma_{B}\right)=\frac{N_{B}}{2 \pi T^{3}}\left[\left(\left(1+x_{6}^{B}\right)-\frac{\left|\lambda_{B}\right|\left(4-\left|\lambda_{B}\right|\right)}{3\left(2-\left|\lambda_{B}\right|\right)^{2}}\right) \lambda_{B}^{2} \sigma_{B}^{3}+2 b_{4} \lambda_{B} \sigma_{B}^{2}+m_{B}^{2} \sigma_{B}\right] .
$$

The quantum effective potential for the field $(\bar{\phi} \phi)_{\mathrm{cl}}$ is related to the above free energies as

$$
U_{\text {eff }}\left((\bar{\phi} \phi)_{\mathrm{cl}}\right)=T^{3} F\left(\sigma_{B}\right) \quad \text { with the replacement } \quad \sigma_{B} \rightarrow \frac{2 \pi(\bar{\phi} \phi)_{\mathrm{cl}}}{N_{B}} .
$$

We continue to use the variable $\sigma_{B}$ as the argument of the effective potential $U_{\text {eff }}$ to avoid clutter, with the understanding that all instances of $\sigma_{B}$ in $U_{\text {eff }}$ are to be replaced with $2 \pi(\bar{\phi} \phi)_{\mathrm{cl}} / N_{B}$. Explicitly, we have

$$
\begin{aligned}
U_{\mathrm{eff}}\left(\sigma_{B}\right)= \begin{cases}\frac{N_{B}}{2 \pi}\left[\left(x_{6}-\phi_{2}\right) \lambda_{B}^{2} \sigma_{B}^{3}+2 \lambda_{B} b_{4} \sigma_{B}^{2}+m_{B}^{2} \sigma_{B}\right] & \text { for } \sigma_{B}<0, \\
\frac{N_{B}}{2 \pi}\left[\left(x_{6}-\phi_{1}\right) \lambda_{B}^{2} \sigma_{B}^{3}+2 \lambda_{B} b_{4} \sigma_{B}^{2}+m_{B}^{2} \sigma_{B}\right] & \text { for } \sigma_{B}>0,\end{cases} \\
\text { with the replacement } \quad \sigma_{B} \rightarrow \frac{2 \pi(\bar{\phi} \phi)_{\mathrm{cl}}}{N_{B}} .
\end{aligned}
$$

The constants $\phi_{1}$ and $\phi_{2}$ are given by

$$
\phi_{1}=\frac{4}{3}\left(\frac{1}{\left(2-\left|\lambda_{B}\right|\right)^{2}}-1\right), \quad \phi_{2}=\frac{4}{3}\left(\frac{1}{\lambda_{B}^{2}}-1\right) .
$$


Observe that the effective potential (5.10) is bounded from below for positive values of $\sigma_{B}$ if the coefficient of $\sigma_{B}^{3}$ is positive in the second of (5.10), i.e. when

$$
x_{6}>\phi_{1} .
$$

Similarly, the effective potential is bounded from below for negative values of $\sigma_{B}$ if the coefficient of the $\sigma_{B}^{3}$ term is negative in the first of (5.10), i.e. when

$$
x_{6}<\phi_{2} .
$$

Note that $\phi_{1}<\phi_{2}$.

Note that the terms proportional to $\sigma_{B}^{2}$ and $\sigma_{B}$ are identical for the two ranges of $\sigma_{B}$ but the coefficients of the $\sigma_{B}^{3}$ terms are different: this non-analyticity in the cubic term is what gives a sharp distinction between the Higgsed and unHiggsed branches of the effective potential at zero temperature. When we turn on temperature we expect this non-analyticity to be smoothed out. ${ }^{14}$

We also give a slightly different expression for the Landau-Ginzburg potential in terms of the variable $c_{B}$ which is useful for the analysis of the gap equations as performed in section 4 of [3]. For this purpose, we substitute back the expressions for $\sigma_{B}$ in terms of $c_{B}$ from (5.6):

$$
U_{\mathrm{eff}}\left(c_{B}\right)=\left\{\begin{array}{cl}
\frac{N_{B}}{2 \pi}\left[A_{u} \frac{c_{B}^{3}}{6}+B_{4, u} \frac{c_{B}^{2}}{2}-m_{B}^{2} \frac{c_{B}}{2}\right] & \text { unHiggsed } \\
\frac{N_{B}\left(2-\left|\lambda_{B}\right|\right)}{2 \pi\left|\lambda_{B}\right|}\left[-A_{h} \frac{c_{B}^{3}}{6}-B_{4, h} \frac{c_{B}^{2}}{2}+m_{B}^{2} \frac{c_{B}}{2}\right] & \text { Higgsed } .
\end{array}\right.
$$

Here, $A_{u}, B_{4, u}$ and $A_{h}, B_{4, h}$ are constants defined by

$$
\begin{array}{ll}
A_{u}=1-\left(1+\frac{3 x_{6}}{4}\right) \lambda_{B}^{2}, & B_{4, u}=\lambda_{B} b_{4}, \\
A_{h}=1-\left(1+\frac{3 x_{6}}{4}\right)\left(2-\left|\lambda_{B}\right|\right)^{2}, & B_{4, h}=-\operatorname{sgn}\left(\lambda_{B}\right)\left(2-\left|\lambda_{B}\right|\right) b_{4} .
\end{array}
$$

The off-shell variable $c_{B}$ has the following advantage; its on-shell value coincides with the pole mass (the gap) of the fundamental excitation in the corresponding phase. We record the gap equations that follow from extremizing (5.14) w.r.t the variable $c_{B}$ :

$$
\begin{aligned}
\text { unHiggsed : } & A_{u} c_{B}^{2}+2 B_{4, u} c_{B}-m_{B}^{2}=0, \\
\text { Higgsed : } & A_{h} c_{B}^{2}+2 B_{4, h} c_{B}-m_{B}^{2}=0 .
\end{aligned}
$$

Solutions to the quadratic equations in (5.16) above correspond to candidates for the Higgsed or unHiggsed phases of the theory. The very recent paper [3] analysed the solutions of (5.16) in detail and used information about the free energy at these solutions to obtain the phase structure as a function of the parameters $x_{6}, \lambda_{B} b_{4}$ and $m_{B}^{2}$. In the next subsection we will extract the same physical information from the exact Landau-Ginzburg effective potential (5.10).

\footnotetext{
${ }^{14}$ This should be easy to verify — and seems to follow from the fact that the finite temperature free energy is an analytic function of its variables - but we have not verified it in detail.
} 


\subsection{Higgsed branch}

In this subsection we study the effective potential (5.10) in more detail on the Higgsed branch.

\subsubsection{Potential for the Higgs vev}

We have already noted around (4.6) that the variable $\sigma_{B}$ has a simple interpretation in terms of the Higgs vev on the Higgsed branch. Making the replacement (4.6), i.e. $\sigma_{B}=$ $2 \pi v^{2} /\left|\lambda_{B}\right|$ in the second line of (5.10) we find

$$
U_{\text {eff }}(v)=\frac{N_{B}}{\left|\lambda_{B}\right|}\left(m_{B}^{2} v^{2}+4 \pi \operatorname{sgn}\left(\lambda_{B}\right) b_{4} v^{4}+\left(\left(1+x_{6}^{B}\right)-\frac{\left|\lambda_{B}\right|\left(4-\left|\lambda_{B}\right|\right)}{3\left(2-\left|\lambda_{B}\right|\right)^{2}}\right) 4 \pi^{2} v^{6}\right),
$$

It is easily verified that (5.17) may also be obtained by taking the zero temperature limit of (3.17) (i.e. dropping the last line in that formula) and integrating $c_{B}$ out of that equation. It follows that the true value of the Higgs vev in the vacuum is obtained by extremizing (5.17).

Note that (5.17) manifestly reduces to the classical potential

$$
U_{\mathrm{cl}}(v)=\frac{N_{B}}{\left|\lambda_{B}\right|}\left(m_{B}^{2} v^{2}+4 \pi \operatorname{sgn}\left(\lambda_{B}\right) b_{4} v^{4}+(2 \pi)^{2}\left(x_{6}+1\right) v^{6}\right),
$$

in the classical limit (2.10) (i.e. $\lambda_{B} \rightarrow 0$ with $m_{B}^{2}, b_{4}$ and $x_{6}$ fixed) as expected on general grounds.

\subsubsection{Graphs of $U_{\text {eff }}\left(\sigma_{B}\right)$ in various cases}

In this subsubsection we will study the graphs of $U_{\text {eff }}\left(\sigma_{B}\right)$ on the Higgsed branch for various ranges of values of microscopic parameters. The results of this subsubsection will prove useful in sketching the phase diagram of the RB theory in later subsections.

Recall that, on the Higgsed branch, $U_{\text {eff }}\left(\sigma_{B}\right)$ is given by the expression

$$
U_{\text {eff }}\left(\sigma_{B}\right)=\frac{N_{B}}{2 \pi}\left(-\frac{\left|\lambda_{B}\right|^{2}}{\left(2-\left|\lambda_{B}\right|\right)^{2}} A_{h} \frac{4 \sigma_{B}^{3}}{3}-\frac{\left|\lambda_{B}\right|}{\left(2-\left|\lambda_{B}\right|\right)} 2 B_{4, h} \sigma_{B}^{2}+m_{B}^{2} \sigma_{B}\right), \quad \sigma_{B}>0,
$$

$\left(A_{h}, B_{4, h}\right.$ were defined in the second line of (5.15)). The extremization of $U_{\text {eff }}\left(\sigma_{B}\right)$ produces the gap equation in the second of (5.16) which we reproduce here:

$$
A_{h} c_{B}^{2}+2 B_{4, h} c_{B}-m_{B}^{2}=0 .
$$

The structure of the curves for $U_{\text {eff }}$ above turn out to depend sensitively on discriminant $D_{h}$ of this gap equation (5.20):

$$
D_{h}=4\left(B_{4, h}^{2}+m_{B}^{2} A_{h}\right) .
$$

As discussed below (5.10), the above effective potential is bounded below when $x_{6}>\phi_{1}$ where $\phi_{1}$ was defined in (5.11). In other words $A_{h}<0$ when $x_{6}>\phi_{1}$ and $A_{h}>0$ when $x_{6}<\phi_{1} \cdot{ }^{15}$

\footnotetext{
${ }^{15}$ Note that $\phi_{1}$ is an increasing function of $\lambda_{B}$. In particular $\phi_{1}=-1$ for the free theory $\left(\lambda_{B}=0\right)$, whereas for the strongly coupled case $\left(\left|\lambda_{B}\right|=1\right)$ we have $\phi_{1}=0$. The fact that $\phi_{1}$ increases as we increase $\left|\lambda_{B}\right|$ indicates that coupling effects increase the propensity of our theory to develop a runaway instability along the $v$ direction.
} 

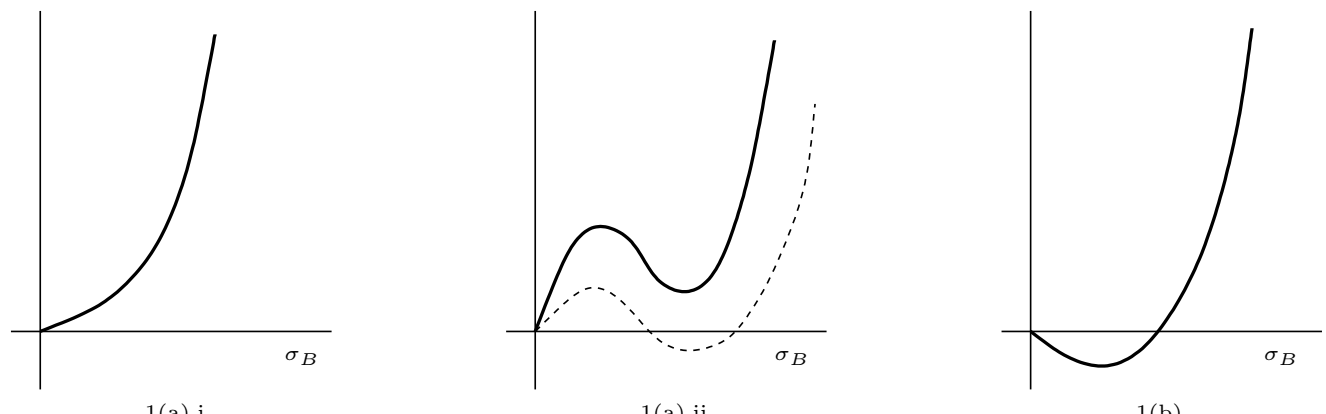

Figure 1. Effective potential in the Higgsed phase for $A_{h}$ negative.

We have the following cases as depicted in figures 1 and 2:

1. $A_{h}$ is negative: the potential $U_{\text {eff }}$ increases at large $\sigma_{B}$.

(a) $m_{B}^{2}$ is positive:

i. $B_{4, h}$ is negative, or $B_{4, h}$ is positive such $D_{h}$ is negative: the potential rises monotonically as $\sigma_{B}$ increases from zero to infinity, and there are no nontrivial positive solutions of the gap equation (5.20).

ii. $B_{4, h}$ is positive such that $D_{h}$ is positive: as $\sigma_{B}$ is increased from zero, $U_{\text {eff }}$ initially increases, reaches a local maximum and then decreases, reaches a minimum and then increases without bound. In this case the gap equation has two solutions; the larger of which is the candidate for a stable phase (the smaller solution presumably describes unstable dynamics since it occurs at a local maximum of the effective potential).

(b) $m_{B}^{2}$ is negative: for either sign of $B_{4, h}, U_{\text {eff }}$ initially decreases, reaches a minimum and then turns and increases indefinitely. The gap equation has exactly one legal solution (i.e. a solution for $c_{B}$ which is positive) which is the candidate for a stable phase.

2. $A_{h}$ is positive: the potential $U_{\text {eff }}$ decreases at large $\sigma_{B}$.

(a) $m_{B}^{2}$ is negative

i. $B_{4, h}$ is positive, or $B_{4, h}$ is negative such that $D_{h}$ is negative: the potential decreases monotonically as $\sigma_{B}$ increases from zero to infinity, and there are no nontrivial positive solutions of the gap equation (5.20).

ii. $B_{4, h}$ is negative such that $D_{h}$ is positive: as $\sigma_{B}$ is increased from zero $U_{\text {eff }}$ initially decreases, reaches a local minimum and then increases till it reaches a local maximum after which it decreases without bound. In this case the gap equation has two solutions; the smaller of which is the candidate metastable phase (the larger solution presumably describes unstable dynamics again since it occurs at a local maximum of the potential). 

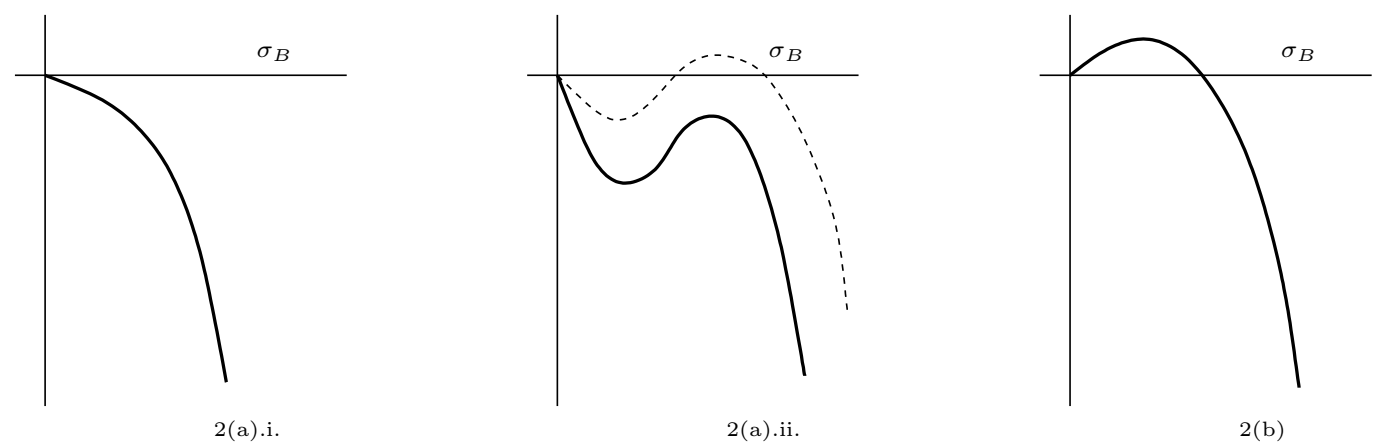

Figure 2. Effective potential in the Higgsed phase for $A_{h}$ negative.

(b) $m_{B}^{2}$ is positive: for either sign of $B_{4, h}, U_{\text {eff }}$ initially increases, reaches a maximum and then turns and decreases indefinitely. The gap equation has exactly one legal solution; this is a local maximum and so presumably describes an unstable 'phase'.

In the last two paragraphs above we have encountered three examples of solutions (1.a.ii, 2.a.ii, 2.b) to the gap equations that describe unstable 'phases'. For future use we note that these three unstable solutions are all given by the following root of (5.20):

$$
c_{B}=\frac{-B_{4, h}+\sqrt{B_{4, h}^{2}+A_{h} m_{B}^{2}}}{A_{h}} .
$$

It is also easy to check that the three cases described above are the only three legal roots of the form (5.22). In other words every local maximum of the potential (5.17) is a root of the form (5.22), and a legal root (i.e. a root for which the r.h.s. is positive) of the form (5.22) is one of the three 'local maxima' situations described above. ${ }^{16}$

\section{3 unHiggsed branch}

\subsubsection{Graphs of $U_{\mathrm{eff}}\left(\sigma_{B}\right)$ in various cases}

In this subsubsection we plot $U_{\text {eff }}\left(\sigma_{B}\right)$ on the unHiggsed branch for various ranges of microscopic parameters.

We start with the following expression for the Landau-Ginzburg potential in the unHiggsed branch in terms of the constants $A_{u}$ and $B_{4, u}$ :

$$
U_{\text {eff }}\left(\sigma_{B}\right)=\frac{N_{B}}{2 \pi}\left(-A_{u} \frac{4 \sigma_{B}^{3}}{3}+2 B_{4, u} \sigma_{B}^{2}+m_{B}^{2} \sigma_{B}\right),
$$

where $A_{u}$ and $B_{4, u}$ are as in the first line of (5.15). Now recall that $\sigma_{B}$ is necessarily negative in the unHiggsed phase. As a consequence (5.23) may be rewritten as

$$
U_{\text {eff }}\left(\sigma_{B}\right)=\frac{N_{B}}{2 \pi}\left(A_{u} \frac{4\left|\sigma_{B}\right|^{3}}{3}+2 B_{4, u}\left|\sigma_{B}\right|^{2}-m_{B}^{2}\left|\sigma_{B}\right|\right) .
$$

\footnotetext{
${ }^{16}$ To repeat, these three cases are as follows. First when $A_{h}$ is negative, $m_{B}^{2}$ positive and $B_{4, h}$ positive such that $D_{h}$ is positive. Second when $A_{h}$ is positive, $m_{B}^{2}$ is negative and $B_{4, h}$ negative such that $D_{h}$ is positive. Lastly when $A_{h}$ is positive and $m_{B}^{2}$ positive for either sign of $B_{4, h}$.
} 

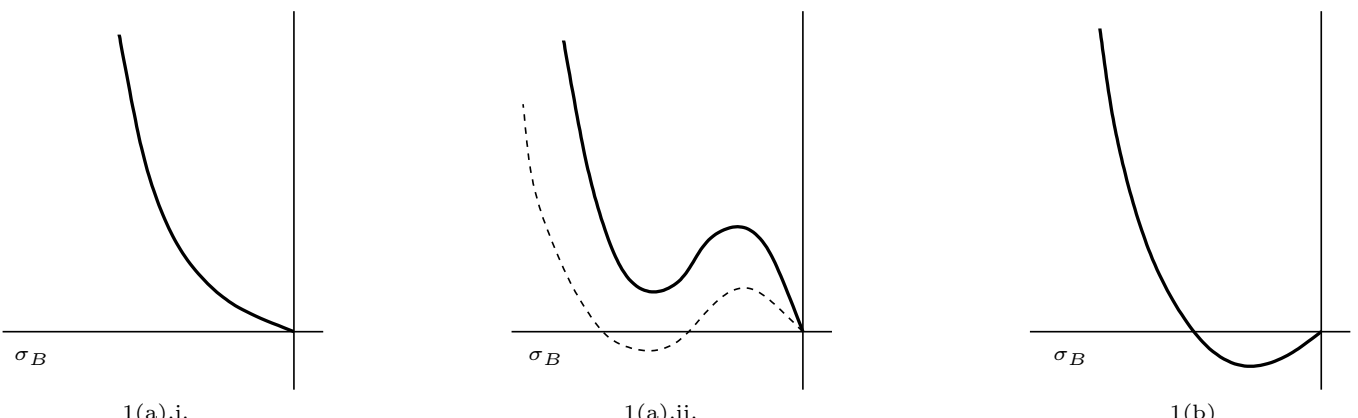

Figure 3. Effective potential in the unHiggsed phase for $A_{u}$ positive.

The gap equation that follows by varying (5.24) w.r.t. $\sigma_{B}$ is given in (5.16) and is reproduced below:

$$
A_{u} c_{B}^{2}+2 B_{4, u} c_{B}-m_{B}^{2}=0,
$$

where $c_{B}=2\left|\sigma_{B}\right|$. Note the formal and notational similarity with the analogous equation (5.20) in the Higgsed phase. As in the previous subsection we briefly analyse the behaviour of (5.24) as a function of $\sigma_{B}$ in all the various cases. We define the discriminant $D_{u}$ of $(5.25)$ :

$$
D_{u}=4\left(B_{4, u}^{2}+m_{B}^{2} A_{u}\right) .
$$

We then have the following cases as depicted in figures 3 and 4 :

1. $A_{u}$ is positive: the potential $U_{\text {eff }}$ increases at large $\left|\sigma_{B}\right|$.

(a) $m_{B}^{2}$ is negative:

i. $B_{4, u}$ is positive, or $B_{4, u}$ is negative such that $D_{u}$ is negative: the potential rises monotonically as $\left|\sigma_{B}\right|$ increases from zero to infinity, and there are no nontrivial positive solutions of the gap equation (5.25).

ii. $B_{4, u}$ is negative such that $D_{u}$ is positive: as $\left|\sigma_{B}\right|$ is increased from zero, $U_{\text {eff }}$ initially increases up to a local maximum and then decreases down to a local minimum and then increases without bound. The gap equation has two solutions the larger of which is the dominant stable phase (the smaller solution presumably describes unstable dynamics since it occurs at a local maximum of the effective potential).

(b) $m_{B}^{2}$ is positive: for either sign of $B_{4, u}, U_{\text {eff }}$ initially decreases, reaches a minimum and then turns and increases indefinitely. The gap equation has exactly one legal solution which is the dominant stable phase.

2. $A_{u}$ is negative: the potential $U_{\text {eff }}$ decreases at large $\left|\sigma_{B}\right|$.

(a) $m_{B}^{2}$ is positive

i. $B_{4, u}$ is negative, or $B_{4, u}$ is positive such that $D_{u}$ is negative: the potential decreases monotonically as $\left|\sigma_{B}\right|$ increases from zero to infinity, and there are no nontrivial positive solutions of the gap equation (5.25). 


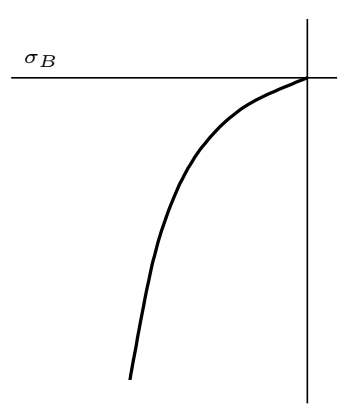

$2(\mathrm{a}) . \mathrm{i}$.

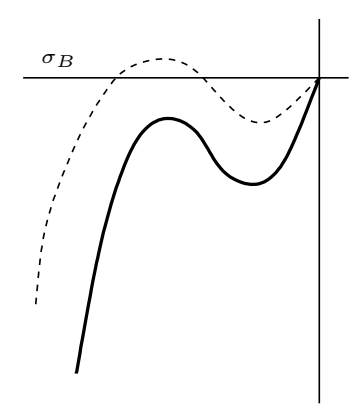

2(a).ii.

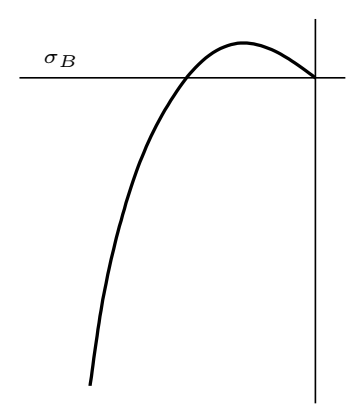

$2(\mathrm{~b})$

Figure 4. Effective potential in the unHiggsed phase for $A_{u}$ negative.

ii. $B_{4, u}$ is positive such that $D_{u}$ is positive: as $\left|\sigma_{B}\right|$ is increased from zero $U_{\text {eff }}$ initially decreases down to a local minimum and then increases up to a local maximum after which it decreases without bound. The smaller of the two solutions to the gap equation (5.25) is the 'dominant' metastable phase (the larger solution presumably describes unstable dynamics again since it occurs at a local maximum of the potential).

(b) $m_{B}^{2}$ is negative: for either sign of $B_{4, u}, U_{\text {eff }}$ initially increases, reaches a maximum and then turns and decreases indefinitely. The gap equation has exactly one legal solution; this is a local maximum and so presumably describes an unstable 'phase'.

In the paragraphs above we have encountered three examples of 'unstable phases' (1.a.ii, 2.a.ii, 2.b). The solution of the gap equation (5.25) associated with each of these phases is easily verified to be

$$
c_{B}=\frac{-B_{4, u}-\sqrt{B_{4, u}^{2}+A_{u} m_{B}^{2}}}{A_{u}} .
$$

Moreover it is also easy to check that every legal (i.e. positive) solution of the form (5.27) is one of the three local maxima of the paragraphs described above.

\subsubsection{Explanation for the instability of local maxima}

We have already explained above that

$$
\left|\sigma_{B}\right|=-\frac{2 \pi(\bar{\phi} \phi)_{\mathrm{cl}}}{N_{B}}
$$

where the r.h.s. of this equation should be interpreted in quantum rather than semiclassical terms since semiclassically the variable $(\bar{\phi} \phi)_{\mathrm{cl}}$ is given by $\langle\bar{\phi}\rangle\langle\phi\rangle$ and is positive. The operator $\bar{\phi} \phi$ however is not necessarily positive (this follows because the subtraction that is used to give this operator meaning is not positive). Equation (5.28) effectively asserts that the unHiggsed branch explores only negative values of the operator $\bar{\phi} \phi$. We have noted above the effective potential as a function of $(\bar{\phi} \phi)_{\mathrm{cl}}$ has unstable 'phases' that sit at local maxima of the effective potential. In the rest of this subsection we will present an explanation of these instabilities. 
Our proposal for the mechanism of the instability of the 'local maxima' phases is that it is the tachyonic instability of a bound state of a single fundamental and antifundamental field in the singlet channel. We claim that the solutions (5.27) are all unstable in this sense, while none of the stable phases - i.e. the phases that occur at legal values of

$$
c_{B}=\frac{-B_{4, u}+\sqrt{B_{4, u}^{2}+A_{u} m_{B}^{2}}}{A_{u}},
$$

suffer from such an instability.

In order to see that this is indeed the case let us recall that bound states do occur as poles in the S-matrix of a fundamental $\phi$ field scattering off an antifundamental $\bar{\phi}$ field. Moreover these poles do sometimes go tachyonic (i.e. their squared mass sometimes goes below zero). The condition for this to happen can be worked out by following discussion in section 4.5 of [5] and appendix $\mathrm{C}$ of [6]. The particle-antiparticle scattering S-matrix has a pole with positive squared mass when

$$
4 \lambda_{B} \leq \lambda_{B}^{2}\left(3 x_{6}^{B}+4\right)-4 \frac{b_{4} \lambda_{B}}{c_{B}} \leq 4 .
$$

When

$$
4 \lambda_{B}=\lambda_{B}^{2}\left(3 x_{6}^{B}+4\right)-4 \frac{b_{4} \lambda_{B}}{c_{B}},
$$

the pole is at threshold, i.e. $\sqrt{s}=2 c_{B}$. On the other hand when

$$
\lambda_{B}^{2}\left(3 x_{6}^{B}+4\right)-4 \frac{b_{4} \lambda_{B}}{c_{B}}=4,
$$

the pole lies at $\sqrt{s}=0$. Using the definitions (5.15) for $A_{u}$ and $B_{4, u}$ it is easy to see that the condition (5.31) can be rewritten as

$$
B_{4, u}=-A_{u} c_{B}
$$

(recall that the quantity $c_{B}$ is positive by definition). When

$$
\lambda_{B}^{2}\left(3 x_{6}^{B}+4\right)-4 \frac{b_{4} \lambda_{B}}{c_{B}}>4,
$$

we have a bound state with negative squared mass, i.e. a tachyonic bound state. The condition for the existence of this tachyonic pole is

$$
A_{u} c_{B} \leq-B_{4, u}
$$

Of course the quantity $c_{B}$ is not independent of $A_{u}$ and $B_{4, u}$ but is determined in terms of these quantities by the gap equation. The solutions to the gap equation are given by

$$
c_{B}=\frac{-B_{4, u} \pm \sqrt{B_{4, u}^{2}+A_{u} m_{B}^{2}}}{A_{u}}
$$


Inserting these solutions into the condition (5.34), we find that the condition (5.34) is met whenever

$$
-B_{4, u} \pm \sqrt{B_{4, u}^{2}+A_{u} m_{B}^{2}} \leq-B_{4, u}
$$

This condition is obeyed by the 'minus' branch of solutions (5.27) but not by the 'plus' branch of solutions (5.29). But we have seen above that this is precisely the split between the local maxima (solutions (5.27)) and local minima (solutions (5.29)) of the effective action (5.24). It is thus natural to identify the tachyonic bound states as the explanation for the instability of the 'minus' branch of solutions (5.27).

It follows, in other words, that the instabilities in the unHiggsed phase occur for the same reason as the instabilities in the Higgsed phase, but for a different field. Unstable Higgsed 'phases' occurred when our solution to the gap equations was at a maximum of the potential for the field $\phi$. We propose that instabilities in the unHiggsed phase occur for solutions to the gap equation around maxima for the field $(\bar{\phi} \phi)_{\mathrm{cl}}$ that is very ostensibly related to the bound state of $\bar{\phi}$ and $\phi$.

In the case of the Higgsed theory in the $\lambda_{B} \rightarrow 0$ limit (2.10) we obtained a classical theory (with an overall factor of $\lambda_{B}^{-1}$ outside the action) in terms of the variable $\varphi$. In the current unHiggsed context the effective potential does not have a clear classical limit as $\lambda_{B} \rightarrow 0$. On solutions to the gap equation that follows from varying (5.24) w.r.t. $\left|\sigma_{B}\right|$, it turns out that $\sigma_{B}$ and $\bar{\phi} \phi$ (rather than $\lambda_{B} \sigma_{B}$ and $\bar{\varphi} \varphi$ as in the Higgsed phase) are finite as $\lambda_{B} \rightarrow 0$. The field $\varphi$ which was the natural classical variable at weak coupling in the Higgsed phase does not seem to be useful in the analysis of the unHiggsed branch (this is probably a reflection of the fact that dynamics is always quantum on this branch).

\subsection{Landau-Ginzburg analysis of the zero temperature phase diagram ${ }^{17}$}

In subsections 5.2 and 5.3 we have already explored the qualitative structure of the LandauGinzburg potential (5.10), plotted as a function of $\sigma_{B}$, separately for $\sigma_{B}>0$ and $\sigma_{B}<0$. In this section we will simply put the analyses of subsections 5.2 and 5.3 together to obtain a global picture of the Landau-Ginzburg potential as a function of $\sigma_{B}$ over all possible ranges of parameters $x_{6}, \lambda_{B} b_{4}$ and $m_{B}^{2}$. We reproduce the potential below. Recall that our exact Landau-Ginzburg potential as a function of $\sigma_{B}$ (or equivalently, using (4.7), a function of $\left.(\bar{\phi} \phi)_{\mathrm{cl}}\right)$ is given by

$$
U_{\mathrm{eff}}\left(\sigma_{B}\right)= \begin{cases}\frac{N_{B}}{2 \pi}\left[\left(x_{6}-\phi_{2}\right) \lambda_{B}^{2} \sigma_{B}^{3}+2 \lambda_{B} b_{4} \sigma_{B}^{2}+m_{B}^{2} \sigma_{B}\right] & \text { for } \sigma_{B}<0 \\ \frac{N_{B}}{2 \pi}\left[\left(x_{6}-\phi_{1}\right) \lambda_{B}^{2} \sigma_{B}^{3}+2 \lambda_{B} b_{4} \sigma_{B}^{2}+m_{B}^{2} \sigma_{B}\right] & \text { for } \sigma_{B}>0 .\end{cases}
$$

Recall from (5.11) that $\phi_{1}<\phi_{2}$. As we have explored in detail above, the plots of the effective potential are qualitatively different when $x_{6}<\phi_{1}, \phi_{1}<x_{6}<\phi_{2}$ and $x_{6}>\phi_{2}$. For this reason we analyse these three ranges of parameters separately. It is also useful to

\footnotetext{
${ }^{17}$ This subsection was worked out in collaboration with O. Aharony.
} 


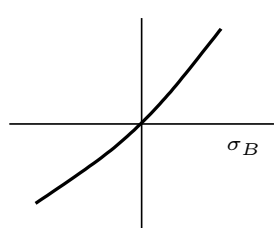

(a)

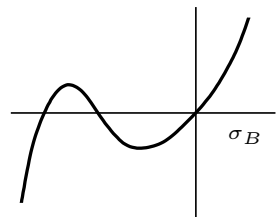

(b)

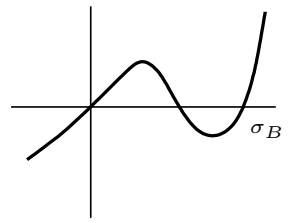

(c)

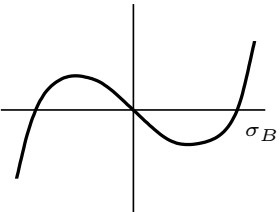

(d)

Figure 5. Effective potential for $x_{6}>\phi_{2}$.

recall the formulae for the discriminants (5.21) and (5.26) of the gap equations (5.16) in either phase of the theory:

$$
\begin{aligned}
& D_{h}=\frac{4\left(2-\left|\lambda_{B}\right|\right)^{2}}{\lambda_{B}^{2}}\left[\left(\lambda_{B} b_{4}\right)^{2}-\frac{3 \lambda_{B}^{2}}{4}\left(x_{6}-\phi_{1}\right) m_{B}^{2}\right], \\
& D_{u}=4\left[\left(\lambda_{B} b_{4}\right)^{2}-\frac{3 \lambda_{B}^{2}}{4}\left(x_{6}-\phi_{2}\right) m_{B}^{2}\right] .
\end{aligned}
$$

\subsubsection{Case I: $x_{6}>\phi_{2}$}

In this case the coefficient of $\sigma_{B}^{3}$ in the effective potential (5.37) is positive both when $\sigma_{B}>0$ and when $\sigma_{B}<0$. It follows that $U_{\text {eff }}\left(\sigma_{B}\right)$ is an increasing function in the limits $\sigma_{B} \rightarrow \pm \infty$. Note, in particular, that $U_{\text {eff }}\left(\sigma_{B}\right)$ is unbounded from below at large negative $\sigma_{B}$ presumably indicating a runaway instability of the theory. In other words, the theory has no truly stable phase in this range of $x_{6}$. In this subsection we will sketch the "phase diagram' of the theory, defined as the diagram that tracks the dominant metastable phase as a function of the relevant parameters. ${ }^{18}$ In order to do this we simply plot $U_{\text {eff }}\left(\sigma_{B}\right)$ as a function of $\sigma_{B}$. The detailed behaviour of the curve $U_{\text {eff }}\left(\sigma_{B}\right)$ at finite values of $\sigma_{B}$ depends on the signs and values of $m_{B}^{2}$ and $\lambda_{B} b_{4}$. We have the following sub cases.

1. $m_{B}^{2}$ positive.

(a) $\lambda_{B} b_{4}$ positive with $D_{u}$ negative or $\lambda_{B} b_{4}$ negative with $D_{h}$ negative: in this case $U_{\text {eff }}\left(\sigma_{B}\right)$ is a monotonically increasing function of $\sigma_{B}$ as depicted in figure $5(\mathrm{a})$. $U_{\text {eff }}\left(\sigma_{B}\right)$ has no extrema and so the gap equation has no solutions.

(b) $\lambda_{B} b_{4}$ positive with $D_{u}$ positive: in this case the curve of $U_{\text {eff }}\left(\sigma_{B}\right)$ takes the schematic form depicted in figure $5(\mathrm{~b})$. $U_{\text {eff }}\left(\sigma_{B}\right)$ has two extrema; a local minimum and a local maximum both for $\sigma_{B}<0$, so both in the unHiggsed branch. The local minimum is the only metastable phase of the theory (the maximum is unstable) and so is the dominant 'phase'.

(c) $\lambda_{B} b_{4}$ negative with $D_{h}$ positive: the graph of $U_{\text {eff }}\left(\sigma_{B}\right)$ takes the schematic form depicted in figure $5(\mathrm{c})$. $U_{\text {eff }}\left(\sigma_{B}\right)$ has two extrema; a local minimum and a local maximum both for positive $\sigma_{B}$ so in the Higgsed branch. The local minimum is the only metastable phase of the theory (the maximum is unstable) and so is the dominant 'phase'.

\footnotetext{
${ }^{18}$ We emphasise that this phase diagram is formal; no phase - not even the dominant one - is stable. The theory always has a run away instability to tunnel to large negative values of $\sigma_{B}$.
} 


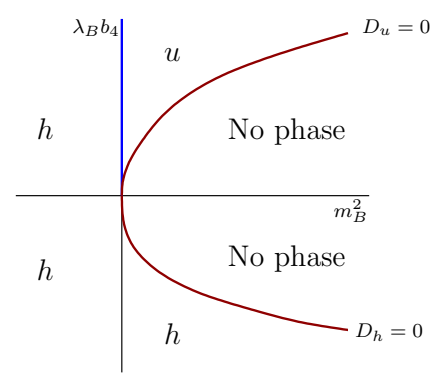

Figure 6. Phase diagram for $x_{6}>\phi_{2}$. The positive $\lambda_{B} b_{4}$ axis (shown in blue) corresponds to a second-order phase transition.

2. $m_{B}^{2}$ negative, $\lambda_{B} b_{4}$ arbitrary: in this case the graph of $U_{\mathrm{eff}}\left(\sigma_{B}\right)$ versus $\sigma_{B}$ takes the schematic form depicted in figure $5(\mathrm{~d})$. We have a local maximum at negative $\sigma_{B}$ (so in the unHiggsed phase) and a local minimum — so a metastable phase - at positive $\sigma_{B}$, so in the Higgsed branch. This local minimum is the dominant (metastable) phase.

Putting all this together we conclude that our theory has the (metastable) phase structure depicted in figure 8 of [3] and redrawn here for convenience in figure 6 of this paper. Notice that whenever a metastable phase exists, a subdominant unstable local maximum of $U_{\text {eff }}\left(\sigma_{B}\right)$ also exists in the vicinity. These are the subdominant 'phases' that appear in figure $22(\mathrm{a})$ of [3].

To end this subsubsection let us study what happens in the limit in which $x_{6} \rightarrow \phi_{2}$ from above. First, nothing special happens to $U_{\text {eff }}\left(\sigma_{B}\right)$ for positive $\sigma_{B}$. At negative $\sigma_{B}$, however, the coefficient of the $\sigma_{B}^{3}$ term tends to zero when $\sigma_{B}<0$. In this limit $D_{u}$ is always positive, so the top half of the red curve in figure 6 tends to a horizontal line (the $m_{B}^{2}>0$ axis). Moreover, when $m_{B}^{2}$ and $\lambda_{B} b_{4}$ are both positive (i.e. the case of figure $5(\mathrm{~b})$ ) the local minimum (which can be thought of as arising due to a competition between the linear and quadratic terms in the action) continues to occur at a fixed value of $\sigma_{B}$ and the $U_{\text {eff }}\left(\sigma_{B}\right)$ evaluated at this minimum also remains fixed. But the local maximum of this diagram (which is a result of the competition between the cubic and quadratic terms in the action) now occurs at a value of $\sigma_{B}$ that tends to $-\infty$. Moreover the value of $U_{\text {eff }}\left(\sigma_{B}\right)$ at this maximum also tends to $\infty$. For $x_{6} \leq \phi_{2}$ this local maximum simply does not exist any more.

\subsubsection{Case II: $\phi_{1}<x_{6}<\phi_{2} \operatorname{phi}(1)=\mathrm{x}(6)=\operatorname{phi}(2)$}

In this case, the coefficient of $\sigma_{B}^{3}$ is positive for $\sigma_{B}>0$ and negative for $\sigma_{B}<0$ which implies that the potential is bounded below for all values of $\sigma_{B}$, so the theory is stable. $U_{\text {eff }}\left(\sigma_{B}\right)$ is a decreasing function of $\sigma_{B}$ for large negative $\sigma_{B}$, but is an increasing function of $\sigma_{B}$ for large positive $\sigma_{B}$.

1. $m_{B}^{2}$ positive: $\lambda_{B} b_{4}$ positive or $\lambda_{B} b_{4}$ negative with $D_{h}$ negative: in this case the graph of $U_{\text {eff }}\left(\sigma_{B}\right)$ versus $\sigma_{B}$ takes the form depicted in figure $7(\mathrm{a})$. The global minimum in the unHiggsed phase (negative $\left.\sigma_{B}\right)$ is the only extremum of $U_{\text {eff }}\left(\sigma_{B}\right)$; this phase dominates the phase diagram. 


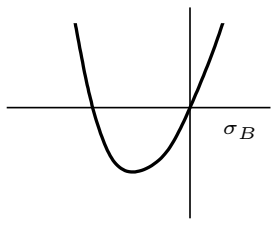

(a)

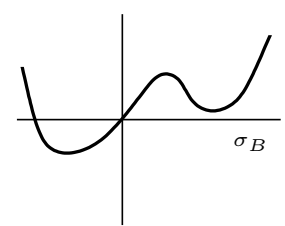

(b)

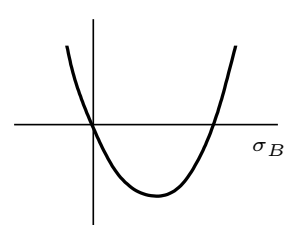

(c)

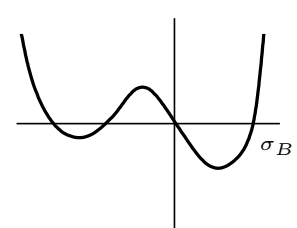

(d)

Figure 7. Effective potential for $\phi_{1}<x_{6}<\phi_{2}$.

2. $m_{B}^{2}$ positive and $\lambda_{B} b_{4}$ negative with $D_{h}$ positive or

$m_{B}^{2}$ negative and $\lambda_{B} b_{4}$ negative with $D_{u}$ positive: in this case the graph of $U_{\mathrm{eff}}\left(\sigma_{B}\right)$ versus $\sigma_{B}$ takes the form depicted in figure $7(\mathrm{~b})$ when $m_{B}^{2}$ is positive and of the form depicted in figure $7(\mathrm{~d})$ when $m_{B}^{2}$ is negative. In either case the graph has a local minimum in the unHiggsed branch (negative $\sigma_{B}$ ) and a local minimum in the Higgsed branch (positive $\sigma_{B}$ ) separated by a local maximum. The maximum occurs in the Higgsed branch when $m_{B}^{2}>0$ but in the unHiggsed branch when $m_{B}^{2}<0 .{ }^{19}$ The dominant phase is the local minimum with the smaller free energy. Which phase dominates depends on the precise values of $m_{B}^{2}, \lambda_{B} b_{4}$ and $x_{6}$. A detailed analysis has been performed in [3] and we summarise the results here. When $x_{6}$ is strictly between $\phi_{1}$ and $\phi_{2}$ the theory has a first order phase transition line along the curve

$$
D_{\nu}=m_{B}^{2}-\nu_{c}\left(x_{6}\right)\left(\lambda_{B} b_{4}\right)^{2}=0 .
$$

The function $\nu_{c}\left(x_{6}\right)$ was studied in detail in [3]; see around figure 25 and figure 26. The function $\nu_{c}\left(x_{6}\right)$ is monotonically decreasing as a function of $x_{6}$ with $x_{6} \in\left(\phi_{1}, \phi_{2}\right)$. The function $\nu_{c}\left(x_{6}\right)$ is negative when $x_{6}$ is near $\phi_{2}$ and hence the first order transition line is in the third quadrant (corresponding to figure $8(\mathrm{a})$ ). When $x_{6}$ is near $\phi_{1}$, the function $\nu_{c}\left(x_{6}\right)$ is positive and hence the first order transition line is in the fourth quadrant (figure $8(\mathrm{~b})$ ). The phase transition line crosses over to the fourth quadrant from the third quadrant (equivalently, $\nu_{c}\left(x_{6}\right)$ goes from being negative to positive) at some intermediate value of $x_{6}$. This intermediate value occurs at $x_{6}=\frac{1}{2}\left(\phi_{1}+\phi_{2}\right)$ and the phase transition line coincides with the negative $\lambda_{B} b_{4}$ axis. We plot the phase diagram for this case and also the corresponding Landau-Ginzburg potential on the phase transition line in figure 9. Clearly, we have two exactly equal minima and hence the onset of a first order phase transition.

Let us study the behaviour in the limits $x_{6} \rightarrow \phi_{1}$ and $x_{6} \rightarrow \phi_{2}$. In the limit $x_{6} \rightarrow \phi_{2}$ from below, the unHiggsed branch minimum occurs at $\sigma_{B} \rightarrow-\infty$ and the potential $U_{\text {eff }}\left(\sigma_{B}\right)$ evaluated on this solution tends to $-\infty$. In this limit the unHiggsed branch local minimum is the dominant phase for every value of $\lambda_{B} b_{4}<0$ and $m_{B}^{2}$. In the opposite limit $x_{6} \rightarrow \phi_{1}$ the Higgsed branch local minimum occurs at very large values of $\sigma_{B}$ and $U_{\text {eff }}\left(\sigma_{B}\right)$ evaluated on this solution tends to $-\infty$. In this limit the Higgsed branch local minimum is the dominant phase for every value of $\lambda_{B} b_{4}<0$ and $m_{B}^{2}$.

\footnotetext{
${ }^{19}$ In fact at $m_{B}^{2}=0$ the local maximum goes through $\sigma_{B}=0$; this maximum undergoes a 'second order phase transition' at this point from the Higgsed to the unHiggsed phase. This point is depicted in figure 9.
} 


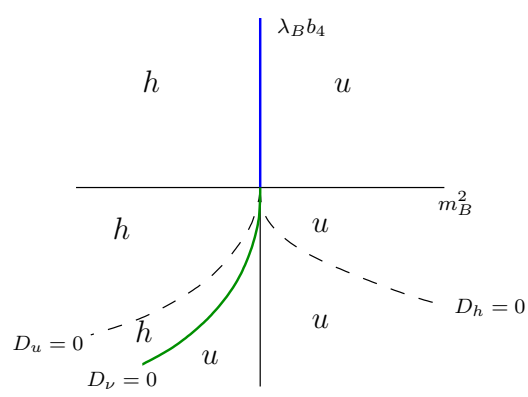

(a)

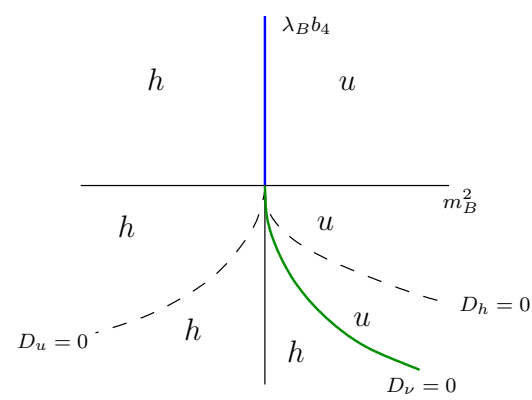

(b)

Figure 8. The phase diagram for $\phi_{1}<x_{6}<\phi_{2}$. There is a second order phase transition (shown in blue) along the positive $\lambda_{B} b_{4}$ axis. The first order phase transition line is the curve (shown in green) between the two dashed curves. The precise location of this phase transition curve varies as we change $x_{6}$. Two possible locations of this curve have been sketched in the two figures above. The first figure corresponds to $x_{6}$ near $\phi_{2}$ and the second figure corresponds to $x_{6}$ near $\phi_{1}$.
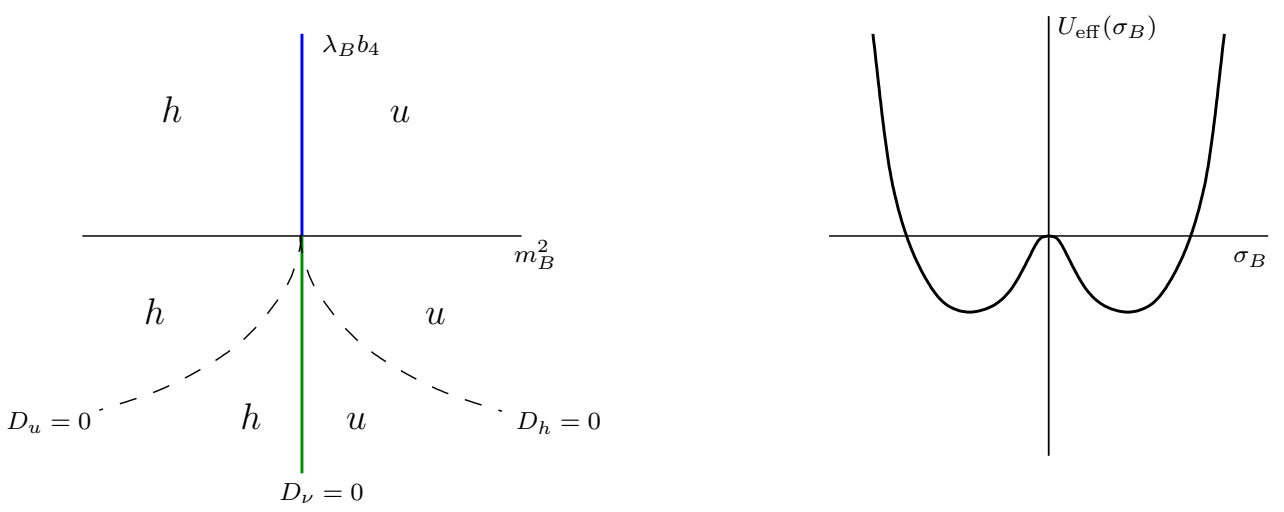

Figure 9. The first figure is the phase diagram for $x_{6}=\frac{1}{2}\left(\phi_{1}+\phi_{2}\right)$. The second figure is the Landau-Ginzburg potential at the same value of $x_{6}$ for a point on the first order phase transition line (green) corresponding to $m_{B}^{2}=0$ and some $\lambda_{B} b_{4}<0$.

3. $m_{B}^{2}$ negative: $\lambda_{B} b_{4}$ positive or $\lambda_{B} b_{4}$ negative with $D_{u}$ negative: the graph of $U_{\text {eff }}\left(\sigma_{B}\right)$ versus $\sigma_{B}$ takes the form depicted in figure $7(\mathrm{c})$. The global minimum in the Higgsed branch (positive $\sigma_{B}$ ) is the only extremum of $U_{\text {eff }}\left(\sigma_{B}\right)$; this phase dominates the phase diagram.

Putting all this together we arrive at the phase diagram presented in figure 7 of [3]. This phase diagram is resketched in figure 8 for convenience.

\subsubsection{Case III: $x_{6}<\phi_{1}=\operatorname{phi}(1)$}

In this case, the coefficient of $\sigma_{B}^{3}$ is negative for both $\sigma_{B}>0$ and $\sigma_{B}<0$. It follows that $U_{\text {eff }}\left(\sigma_{B}\right)$ is a increasing function in the limits $\sigma_{B} \rightarrow \pm \infty$. Note, in particular, that $U_{\text {eff }}\left(\sigma_{B}\right)$ is unbounded from below at large positive $\sigma_{B}$ presumably implying a runaway instability of the theory. In this case the instability is easy to understand as it is present even in the classical theory at sufficiently negative values of $x_{6}$. Just as in section 5.4.1, in 


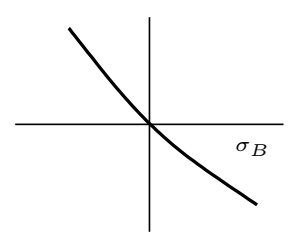

(a)

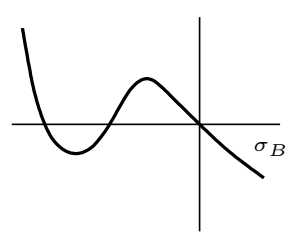

(b)

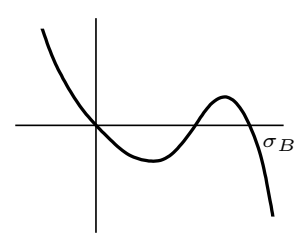

(c)

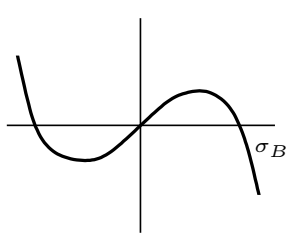

(d)

Figure 10. Effective potential for $x_{6}<\phi_{1}$.

range of parameters the RB the theory has no truly stable phases. As in section 5.4.1, in this subsubsection we will sketch the 'phase diagram' of the theory, defined as the diagram that tracks the dominant metastable phase as a function of relevant parameters. As in section 5.4.1 we read off our results from plots of $U_{\text {eff }}\left(\sigma_{B}\right)$ as a function of $\sigma_{B}$. We have the following subcases.

1. $m_{B}^{2}$ negative.

(a) $\lambda_{B} b_{4}$ negative with $D_{u}$ negative or $\lambda_{B} b_{4}$ positive with $D_{h}$ negative: $U_{\text {eff }}\left(\sigma_{B}\right)$ is a monotonically decreasing function of $\sigma_{B}$ as depicted in figure 10(a). $U_{\text {eff }}\left(\sigma_{B}\right)$ has no extrema, and so the gap equation has no solutions.

(b) $\lambda_{B} b_{4}$ negative with $D_{u}$ positive: in this case the curve of $U_{\text {eff }}\left(\sigma_{B}\right)$ takes the schematic form depicted in figure 10(b). $U_{\text {eff }}\left(\sigma_{B}\right)$ has two extrema; a local minimum and a local maximum both for $\sigma_{B}<0$, so both in the unHiggsed phase. The local minimum is the only metastable phase of the theory (the maximum is unstable) and so is the dominant 'phase'.

(c) $\lambda_{B} b_{4}$ positive with $D_{h}$ positive: the graph of $U_{\text {eff }}\left(\sigma_{B}\right)$ takes the schematic form depicted in figure $10(\mathrm{c}) . U_{\text {eff }}\left(\sigma_{B}\right)$ has two extrema; a local minimum and a local maximum both for positive $\sigma_{B}$ so in the Higgsed phase. The local minimum is the only metastable phase of the theory (the maximum is unstable) and so is the dominant 'phase'.

2. $m_{B}^{2}$ positive, $\lambda_{B} b_{4}$ arbitrary: in this case the graph of $U_{\text {eff }}\left(\sigma_{B}\right)$ versus $\sigma_{B}$ takes the form depicted in figure $10(\mathrm{~d})$. We have a local minimum in at negative $\sigma_{B}$ (so in the unHiggsed phase) and a local maximum at positive $\sigma_{B}$, so in the Higgsed phase. This local minimum is the dominant (metastable) phase.

Putting all this together we conclude that our theory has the (metastable) phase structure depicted in figure 9 of [3] and redrawn here for convenience in figure 11 of this paper.

Notice that whenever a metastable phase exists, a subdominant unstable local maximum of $U_{\text {eff }}\left(\sigma_{B}\right)$ also exists in the vicinity. These are the subdominant 'phases' that appear in figure $27(\mathrm{~b})$ of [3].

To end this subsubsection let us study what happens in the limit in which $x_{6} \rightarrow \phi_{1}$ from above. For negative values of $\sigma_{B}$, nothing special happens to $U_{\text {eff }}\left(\sigma_{B}\right)$. At positive $\sigma_{B}$, however, the coefficient of the $\sigma_{B}^{3}$ term tends to zero. In this limit $D_{h}$ is always positive, so the top half of the red curve in figure 11 tends to a horizontal line (the negative $m_{B}^{2}$ 


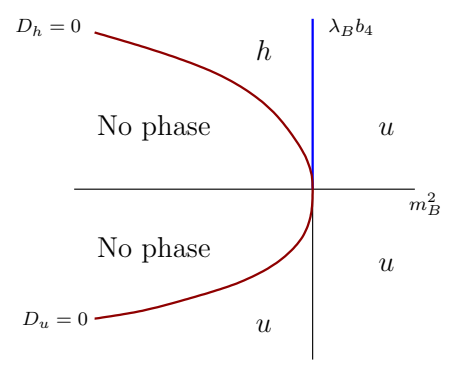

Figure 11. Phase diagram for $x_{6}<\phi_{1}$. The positive $\lambda_{B} b_{4}$ axis (shown in blue) corresponds to a second-order phase transition.

axis). Moreover, when $m_{B}^{2}$ and $\lambda_{B} b_{4}$ are both positive (i.e. the case of sub figure $10(\mathrm{~d})$ ) the local minimum (which can be thought of as arising due to a competition between the linear and quadratic terms in the action) continues to occur at a fixed value of $\sigma_{B}$ and the $U_{\text {eff }}\left(\sigma_{B}\right)$ evaluated at this minimum also remains fixed. But the local maximum of this diagram (which is a result of the competition between the cubic and quadratic terms in the action) now occurs at a value of $\sigma_{B}$ that tends to $\infty$. Moreover the value of $U_{\text {eff }}\left(\sigma_{B}\right)$ at this maximum also tends to $\infty$. For $x_{6} \geq \phi_{1}$ this local maximum simply does not exist any more.

\section{Discussion}

The results of this paper suggest several questions for future work. First, it would be interesting to generalise the computation of S-matrices presented in $[5,6]$ to the Higgsed phase of the Regular Boson theory. The fact that this (and related) computations may throw light on the dual fermionic interpretation of the $Z$ boson - as discussed in detail in [1] - make it particularly interesting.

One of the most interesting results of this paper is the off-shell effective action (5.10). It would be interesting to generalise this result to finite values of temperature and chemical potential and explicitly observe the smoothing-out of the non-analyticity which was present at zero temperature. It would be also interesting to compute a similar action for the theory of one fundamental boson and one fundamental fermion studied in [34] and to use this action to unravel the phase structure of the deformed $\mathcal{N}=2$ supersymmetric matter Chern-Simons theory with a single chiral multiplet in the fundamental representation. It is possible that such an investigation will have interesting interplays with supersymmetry: for example it may be possible to find a superspace version of (5.10).

In section 4 we have presented a three-variable off-shell free energy that reproduces the gap equation and thermal free energy of the regular boson theory. We have also presented a physical interpretation of the variable $\sigma_{B}$ that enters this action. It would be interesting to investigate whether there are interesting off-shell interpretations of the other dynamical variables $-c_{B}$ and $\tilde{\mathcal{S}}$ - that appear in section 4 .

Above, we have found a preferred value for the cosmological constant counterterm $\Lambda$ of the $\mathrm{CB}$ theory - one that correctly reproduces the tadpole condition for the regular boson 
theory (see (3.19)). It would be interesting to derive (3.19) from a more fundamental physical principle. It would also be interesting to investigate if this result makes any physical predictions for the critical boson theory: is it correct, for instance, to interpret the Legendre transform of (3.13) w.r.t. $m_{B}^{\text {cri }}$ (with $\Lambda$ chosen to have the value (3.19)) as the Coleman-Weinberg potential of the CB theory w.r.t. its dimension two scalar operator $J_{0}$ ? It would be interesting to further investigate this and similar questions, and their implications.

Finally it would be interesting to generalise the considerations of this paper - even qualitatively — to finite values of $N_{B}$. We leave all these questions for future work.

\section{Acknowledgments}

We would like to thank F. Benini and D. Radicevic for useful discussions. We would especially like to thank O. Aharony for collaboration on section 5.4, for several very useful discussions over the course of many months and for very useful comments on a preliminary version of this manuscript. The work of A. D., I. H., L. J., S. M., and N. P. was supported by the Infosys Endowment for the study of the Quantum Structure of Spacetime. S. J. would like to thank TIFR, Mumbai for hospitality during the completion of the work. The work of S. J. is supported by the Ramanujan Fellowship. Finally we would all like to acknowledge our debt to the steady support of the people of India for research in the basic sciences.

\section{A Previously known results for the large $N$ free energy}

Below we will encounter several equations that involve the quantities

$$
\begin{aligned}
\mathcal{C} & =\frac{1}{2} \int_{-\pi}^{\pi} d \alpha \rho_{F}(\alpha)\left(\log \left(2 \cosh \left(\frac{\hat{c}_{F}+i \alpha}{2}\right)\right)+\log \left(2 \cosh \left(\frac{\hat{c}_{F}-i \alpha}{2}\right)\right)\right), \\
\mathcal{S} & =\frac{1}{2} \int_{-\pi}^{\pi} d \alpha \rho_{B}(\alpha)\left(\log \left(2 \sinh \left(\frac{\hat{c}_{B}+i \alpha}{2}\right)\right)+\log \left(2 \sinh \left(\frac{\hat{c}_{B}-i \alpha}{2}\right)\right)\right),
\end{aligned}
$$

where $\hat{c}_{B}$ and $\hat{c}_{F}$ are (dimensionless versions of) the thermal masses in the boson and fermion theory respectively.

Using (2.14), it is not difficult to verify the following identities:

$$
\lambda_{B} \mathcal{S}=-\frac{\operatorname{sgn}\left(\lambda_{F}\right)}{2} c_{F}+\lambda_{F} \mathcal{C}, \quad \lambda_{F} \mathcal{C}=-\frac{\operatorname{sgn}\left(\lambda_{B}\right)}{2} c_{B}+\lambda_{B} \mathcal{S}
$$

\section{A.1 Results for the critical fermion theory}

In [2] the 'fixed holonomy' $\mathbb{R}^{2} \times S^{1}$ partition function - $v_{F}\left[\rho_{F}\right]$ - of the fermionic theory has been evaluated in both fermionic phases. The final result of this calculation is most 
conveniently given in terms of an auxiliary off-shell free energy

$$
\begin{aligned}
F_{F}\left(c_{F}, \zeta_{F}, \tilde{\mathcal{C}}\right) \\
=\frac{N_{F}}{6 \pi}\left[\hat{c}_{F}^{3}-2 \lambda_{F}^{2} \tilde{\mathcal{C}}^{3}-\frac{3}{2}\left(\hat{c}_{F}^{2}-\frac{16 \pi^{2}}{\kappa_{F}^{2}} \hat{\zeta}_{F}^{2}\right) \tilde{\mathcal{C}}+\frac{6 \pi \hat{y}_{2}^{2}}{\kappa_{F} \lambda_{F}} \hat{\zeta}_{F}-\frac{24 \pi^{2} \hat{y}_{4}}{\kappa_{F}^{2} \lambda_{F}} \hat{\zeta}_{F}^{2}+\frac{24 \pi^{3} x_{6}^{F}}{\kappa_{F}^{3} \lambda_{F}} \hat{\zeta}_{F}^{3}\right. \\
\quad-\frac{3}{2} \tilde{\mathcal{C}}\left(\hat{c}_{F}^{2}-\left(2 \lambda_{F} \tilde{\mathcal{C}}-\frac{4 \pi}{\kappa_{F}} \hat{\zeta}_{F}\right)^{2}\right) \\
\left.\quad-3 \int_{-\pi}^{\pi} d \alpha \rho_{F}(\alpha) \int_{\hat{c}_{F}}^{\infty} d y y\left(\log \left(1+e^{-y-i \alpha}\right)+\log \left(1+e^{-y+i \alpha}\right)\right)\right] .
\end{aligned}
$$

The auxiliary off-shell free energy (A.3) is a function of three variables $-c_{F}, \zeta_{F}$ and $\tilde{\mathcal{C}}$ - in addition to the temperature and the holonomies. The free energy $v_{F}\left[\rho_{F}\right]$ defined in (2.11) is obtained from $F_{F}\left[\rho_{F}\right]$ in (A.3) by extremizing the latter quantity w.r.t. these three 'dynamical' variables. Extremizing the free energy (A.3) w.r.t. the variable $\tilde{\mathcal{C}}$ yields the equation of motion

$$
\hat{c}_{F}^{2}=\left(2 \lambda_{F} \tilde{\mathcal{C}}-\frac{4 \pi}{\kappa_{F}} \hat{\zeta}_{F}\right)^{2}
$$

Varying w.r.t. $c_{F}$ yields

$$
\tilde{\mathcal{C}}=\mathcal{C}
$$

with $\mathcal{C}$ given in (A.1), while the stationarity of variation w.r.t. $\zeta_{F}$ yields

$$
-\frac{3}{4}\left(\frac{4 \pi \hat{\zeta}_{F}}{\kappa_{F}}\right)^{2} x_{6}^{F}-\frac{16 \pi \hat{\zeta}_{F}}{\kappa_{F}} \lambda_{F} \tilde{\mathcal{C}}+\frac{8 \pi \hat{\zeta}_{F}}{\kappa_{F}} \hat{y}_{4}+4 \lambda_{F}^{2} \tilde{\mathcal{C}}^{2}-\hat{y}_{2}^{2}=0 .
$$

Plugging (A.5) into (A.4) and (A.6) respectively yields the simplified gap equations

$$
\hat{c}_{F}^{2}=\left(2 \lambda_{F} \mathcal{C}-\frac{4 \pi \hat{\zeta}_{F}}{\kappa_{F}}\right)^{2},
$$

and

$$
-\frac{3}{4}\left(\frac{4 \pi \hat{\zeta}_{F}}{\kappa_{F}}\right)^{2} x_{6}^{F}-\frac{16 \pi \hat{\zeta}_{F}}{\kappa_{F}} \lambda_{F} \mathcal{C}+\frac{8 \pi \hat{\zeta}_{F}}{\kappa_{F}} \hat{y}_{4}+4 \lambda_{F}^{2} \mathcal{C}^{2}-\hat{y}_{2}^{2}=0,
$$

for the quantities $c_{F}$ and $\zeta_{F}$.

Below we will find it useful to work with a reduced off-shell free energy, obtained by integrating $\tilde{\mathcal{C}}$ out of (A.3). In order to do this we note that (A.4) has two solutions

$$
2 \lambda_{F} \tilde{\mathcal{C}}=\frac{4 \pi \hat{\zeta}_{F}}{\kappa_{F}} \pm \hat{c}_{F}
$$

The undetermined sign in (A.9) is completely free. Clearly this sign is (tautologically) given by $\operatorname{sgn}\left(\tilde{X}_{F}\right)$ where

$$
\tilde{X}_{F} \equiv 2 \lambda_{F} \tilde{\mathcal{C}}-\frac{4 \pi \hat{\zeta}_{F}}{\kappa_{F}} .
$$

It follows that (A.9) may formally be rewritten as

$$
2 \lambda_{F} \tilde{\mathcal{C}}=\frac{4 \pi \hat{\zeta}_{F}}{\kappa_{F}}+\operatorname{sgn}\left(\tilde{X}_{F}\right) \hat{c}_{F} .
$$


The reduced free energy — which is now a function only of two variables $c_{F}$ and $\zeta_{F}$ - is obtained by plugging either of these two solutions into (A.3). Note that when we do this the second line of (A.3) vanishes as a consequence of (A.4). ${ }^{20}$ Inserting the solutions (A.11) into the free energy (A.3), we have the following explicit expression for the reduced off-shell free energy as a function of $c_{F}$ and $\zeta_{F}$

$$
\begin{aligned}
F_{F}\left(c_{F}, \zeta_{F}\right)= & \frac{N_{F}}{6 \pi}\left[\frac{\left|\lambda_{F}\right|-\operatorname{sgn}\left(\lambda_{F}\right) \operatorname{sgn}\left(\tilde{X}_{F}\right)}{\left|\lambda_{F}\right|} \hat{c}_{F}^{3}-\frac{3}{2 \lambda_{F}}\left(\frac{4 \pi \hat{\zeta}_{F}}{\kappa_{F}}\right)\left(\hat{c}_{F}^{2}-\hat{y}_{2}^{2}\right)\right. \\
& -\frac{3 \hat{y}_{4}}{2 \lambda_{F}}\left(\frac{4 \pi \hat{\zeta}_{F}}{\kappa_{F}}\right)^{2}+\frac{\left(3 x_{6}^{F}+4\right)}{8 \lambda_{F}}\left(\frac{4 \pi \hat{\zeta}_{F}}{\kappa_{F}}\right)^{3} \\
& \left.-3 \int_{-\pi}^{\pi} d \alpha \rho_{F}(\alpha) \int_{\hat{c}_{F}}^{\infty} d y y\left(\log \left(1+e^{-y-i \alpha}\right)+\log \left(1+e^{-y+i \alpha}\right)\right)\right] .
\end{aligned}
$$

Note that the above reduced off-shell free energy function has two branches depending on the $\operatorname{sign} \operatorname{sgn}\left(\tilde{X}_{F}\right)$. We refer to the branch in which $\operatorname{sgn}\left(\tilde{X}_{F}\right) \operatorname{sgn}\left(\lambda_{F}\right)>0$ as the unHiggsed branch, and the branch in which $\operatorname{sgn}\left(\tilde{X}_{F}\right) \operatorname{sgn}\left(\lambda_{F}\right)<0$ as the Higgsed branch. It is easily verified that the variation of (A.12) with respect to $\hat{c}_{F}$ yields (A.7) while the variation of (A.12) w.r.t. $\zeta_{F}$ yields (A.8).

Note that that the gap equations (A.4), (A.5) (equivalently (A.7)) and (A.6) - unlike the free energy (A.12) - have no explicit dependence on $\operatorname{sgn}\left(\tilde{X}_{F}\right)$. Nonetheless the same gap equations hold for both 'phases' of the theory, i.e. for both choices of $\operatorname{sgn}\left(\tilde{X}_{F} \lambda_{F}\right){ }^{21}$ It follows from this observation that the solutions to the finite temperature gap equations of the fermionic theory vary analytically as we pass from one 'phase' to another. In fact more is true; the finite temperature free energy of the fermionic theory is itself analytic as one passes from the unHiggsed to the Higgsed phase. At the physical level, the sharp zero temperature distinction between the Higgsed and unHiggsed phases gets blurred out by finite temperature effects.

\section{A.2 Results for regular bosons in the unHiggsed phase}

The off-shell free energy for the RB theory was computed in [2] and is given by

$$
\begin{aligned}
F_{B}\left(c_{B}, \tilde{\mathcal{S}}\right)= & \frac{N_{B}}{6 \pi}\left[-\hat{c}_{B}^{3}+2\left(\hat{c}_{B}^{2}-\hat{m}_{B}^{2}\right) \tilde{\mathcal{S}}+2 \lambda_{B} \hat{b}_{4} \tilde{\mathcal{S}}^{2}\right. \\
& +\tilde{\mathcal{S}}\left(\hat{c}_{B}^{2}-\hat{m}_{B}^{2}-\left(4+3 x_{6}^{B}\right) \lambda_{B}^{2} \tilde{\mathcal{S}}^{2}+4 \lambda_{B} \hat{b}_{4} \tilde{\mathcal{S}}\right) \\
& \left.+3 \int_{-\pi}^{\pi} d \alpha \rho_{B}(\alpha) \int_{\hat{c}_{B}}^{\infty} d y y\left(\log \left(1-e^{-y-i \alpha}\right)+\log \left(1-e^{-y+i \alpha}\right)\right)\right],
\end{aligned}
$$

\footnotetext{
${ }^{20}$ This reduced form of the off-shell free energy — rather than the fully off-shell free energy (A.3) — was presented in [2]. The off-shell free energy (A.3) is a new formula that has not previously been presented in the literature.

${ }^{21}$ The fact that $\operatorname{sgn}\left(\tilde{X}_{F}\right)$ disappears from the gap equations is obvious when we obtain the equations from (A.3), even though this fact might appear mysterious when derived starting from (A.12).
} 
or equivalently by

$$
\begin{aligned}
F_{B}\left(c_{B}, \tilde{\mathcal{S}}\right)= & \frac{N_{B}}{6 \pi}\left[-\hat{c}_{B}^{3}+3 \tilde{\mathcal{S}}\left(\hat{c}_{B}^{2}-\hat{m}_{B}^{2}\right)+6 \hat{b}_{4} \lambda_{B} \tilde{\mathcal{S}}^{2}-\left(4+3 x_{6}^{B}\right) \lambda_{B}^{2} \tilde{\mathcal{S}}^{3}\right. \\
& \left.+3 \int_{-\pi}^{\pi} d \alpha \rho_{B}(\alpha) \int_{\hat{c}_{B}}^{\infty} d y y\left(\log \left(1-e^{-y-i \alpha}\right)+\log \left(1-e^{-y+i \alpha}\right)\right)\right] .
\end{aligned}
$$

As in the previous subsection, the free energy $v_{B}\left[\rho_{B}\right]$ defined in (2.11) is obtained by extremizing the action (A.13) w.r.t. the dynamical variables $\tilde{\mathcal{S}}$ and $c_{B}$. The equations of motion that follow by varying (A.13) w.r.t. $\tilde{\mathcal{S}}$ and $c_{B}$ respectively are

$$
\hat{c}_{B}^{2}=\left(4+3 x_{6}^{B}\right) \lambda_{B}^{2} \tilde{\mathcal{S}}^{2}-4 \lambda_{B} \hat{b}_{4} \tilde{\mathcal{S}}+\hat{m}_{B}^{2},
$$

and

$$
\tilde{\mathcal{S}}=\mathcal{S}
$$

where $\mathcal{S}$ was defined in (A.1). Inserting (A.16) into (A.15) yields the gap equation for the single variable $c_{B}$

$$
\hat{c}_{B}^{2}=\left(4+3 x_{6}^{B}\right) \lambda_{B}^{2} \mathcal{S}^{2}-4 \lambda_{B} \hat{b}_{4} \mathcal{S}+\hat{m}_{B}^{2}
$$

As in the previous subsection it is possible to obtain a reduced free energy by integrating $\tilde{\mathcal{S}}$ out of (A.13). This may be achieved by using (A.15) to solve for $\tilde{\mathcal{S}}$ as a function of $c_{B}$ and plugging this solution into (A.13). ${ }^{22}$ This is the form in which the off-shell free energy for the scalar theory in the unHiggsed phase was presented in [2].

\section{A.3 Duality in the unHiggsed phase and a prediction for the Higgsed phase}

We first list the duality map between various quantities in the RB and CF theories. Recall that the 't Hooft parameters $\lambda_{B}$ and $\lambda_{F}$ were defined as

$$
\lambda_{B}=\frac{N_{B}}{\kappa_{B}}, \quad \lambda_{F}=\frac{N_{F}}{\kappa_{F}} .
$$

The duality maps different parameters as follows:

$$
\begin{aligned}
& N_{F}=\left|\kappa_{B}\right|-N_{B}, \quad \kappa_{F}=-\kappa_{B}, \quad \lambda_{F}=\lambda_{B}-\operatorname{sgn}\left(\lambda_{B}\right), \\
& x_{6}^{F}=x_{6}^{B}, \quad y_{4}=b_{4}, \quad y_{2}^{2}=m_{B}^{2}, \quad\left|\lambda_{B}\right| \rho_{B}(\alpha)+\left|\lambda_{F}\right| \rho_{F}(\pi-\alpha)=\frac{1}{2 \pi} .
\end{aligned}
$$

The last relation gives rise to

$$
N_{F} \rho_{F}(\alpha)=\frac{\left|\kappa_{B}\right|}{2 \pi}-N_{B} \rho_{B}(\pi-\alpha)
$$

\footnotetext{
${ }^{22}$ Note that the second line of (A.13) vanishes when we do this as a this line is proportional to (A.15).
} 
Consider the off-shell free energies for the critical fermion theory in terms of the two 'fields' $c_{F}$ and $\zeta_{F}$ given in (A.12):

$$
\begin{aligned}
F_{F}\left(c_{F}, \zeta_{F}\right)= & \frac{N_{F}}{6 \pi}\left[\frac{\lambda_{F}-\operatorname{sgn}\left(\tilde{X}_{F}\right)}{\lambda_{F}} \hat{c}_{F}^{3}-\frac{3}{2 \lambda_{F}}\left(\frac{4 \pi \hat{\zeta}_{F}}{\kappa_{F}}\right)\left(\hat{c}_{F}^{2}-\hat{y}_{2}^{2}\right)\right. \\
& -\frac{3 \hat{y}_{4}}{2 \lambda_{F}}\left(\frac{4 \pi \hat{\zeta}_{F}}{\kappa_{F}}\right)^{2}+\frac{\left(3 x_{6}^{F}+4\right)}{8 \lambda_{F}}\left(\frac{4 \pi \hat{\zeta}_{F}}{\kappa_{F}}\right)^{3}+ \\
& \left.-3 \int_{-\pi}^{\pi} d \alpha \rho_{F}(\alpha) \int_{\hat{c}_{B}}^{\infty} d y y\left(\log \left(1+e^{-y-i \alpha}\right)+\log \left(1+e^{-y+i \alpha}\right)\right)\right] .
\end{aligned}
$$

Using the relation (A.20), the last line in (A.21) can be rewritten $\operatorname{as}^{23}$

$$
\begin{aligned}
- & \frac{3 N_{F}}{6 \pi} \int_{-\pi}^{\pi} d \alpha \rho_{F}(\alpha) \int_{\hat{c}_{F}}^{\infty} d y y\left(\log \left(1+e^{-y-i \alpha}\right)+\log \left(1+e^{-y+i \alpha}\right)\right)= \\
= & \frac{3 N_{B}}{6 \pi} \int_{-\pi}^{\pi} d \alpha \rho_{B}(\alpha) \int_{\hat{c}_{F}}^{\infty} d y y\left(\log \left(1-e^{-y-i \alpha}\right)+\log \left(1-e^{-y+i \alpha}\right)\right) .
\end{aligned}
$$

Substituting the various fermionic parameters with their bosonic counterparts in (A.19), we get the following expression for the dual of the fermionic off-shell free energy in terms of two 'fields' $c_{F}$ and $\zeta_{F}$ :

$$
\begin{aligned}
\mathcal{F}\left(c_{F}, \zeta_{F}\right)= & \frac{N_{B}}{6 \pi}\left[-\frac{\lambda_{B}-\operatorname{sgn}\left(\lambda_{B}\right)-\operatorname{sgn}\left(\tilde{X}_{F}\right)}{\lambda_{B}} \hat{c}_{F}^{3}+\frac{3}{2 \lambda_{B}}\left(\frac{4 \pi \hat{\zeta}_{F}}{\kappa_{F}}\right)\left(\hat{c}_{F}^{2}-\hat{m}_{B}^{2}\right)+\right. \\
& +\frac{3 \hat{b}_{4}}{2 \lambda_{B}}\left(\frac{4 \pi \hat{\zeta}_{F}}{\kappa_{F}}\right)^{2}-\frac{\left(3 x_{6}^{B}+4\right)}{8 \lambda_{B}}\left(\frac{4 \pi \hat{\zeta}_{F}}{\kappa_{F}}\right)^{3}+ \\
& \left.+3 \int_{-\pi}^{\pi} d \alpha \rho_{B}(\alpha) \int_{\hat{c}_{F}}^{\infty} d y y\left(\log \left(1-e^{-y-i \alpha}\right)+\log \left(1-e^{-y+i \alpha}\right)\right)\right] .
\end{aligned}
$$

\section{A.3.1 unHiggsed phase: $\operatorname{sgn}\left(X_{F}\right)=-\operatorname{sgn}\left(\lambda_{B}\right)$}

In this case $\mathcal{F}\left(c_{F}, \zeta_{F}\right)$ in (A.23) simplifies to

$$
\begin{aligned}
\mathcal{F}\left(c_{F}, \zeta_{F}\right)= & \frac{N_{B}}{6 \pi}\left[-\hat{c}_{F}^{3}+\frac{3}{2 \lambda_{B}}\left(\frac{4 \pi \hat{\zeta}_{F}}{\kappa_{F}}\right)\left(\hat{c}_{F}^{2}-\hat{m}_{B}^{2}\right)+\right. \\
& +\frac{3 \hat{b}_{4}}{2 \lambda_{B}}\left(\frac{4 \pi \hat{\zeta}_{F}}{\kappa_{F}}\right)^{2}-\frac{\left(3 x_{6}^{B}+4\right)}{8 \lambda_{B}}\left(\frac{4 \pi \hat{\zeta}_{F}}{\kappa_{F}}\right)^{3}+ \\
& \left.+3 \int_{-\pi}^{\pi} d \alpha \rho_{B}(\alpha) \int_{\hat{c}_{F}}^{\infty} d y y\left(\log \left(1-e^{-y-i \alpha}\right)+\log \left(1-e^{-y+i \alpha}\right)\right)\right] .
\end{aligned}
$$

\footnotetext{
${ }^{23}$ The two terms in the r.h.s. of (A.20) simplify as follows. The integral over $\alpha$ in the term proportional to $\left|\kappa_{B}\right|$ can be performed by Taylor-expanding the logarithms and gives zero since the integrals are of the form $\int_{-\pi}^{\pi} d \alpha e^{i n \alpha}=0$ for non-zero integers $n$. In the term proportional to $\rho_{B}(\pi-\alpha)$, we have performed the variable change $\alpha \rightarrow \pi-\alpha$ resulting in an additional minus sign in the argument of the logarithms.
} 
If we now perform the field redefinitions

$$
\hat{c}_{F}=\hat{c}_{B}, \quad \frac{4 \pi \hat{\zeta}_{F}}{\kappa_{F}}=2 \lambda_{B} \tilde{\mathcal{S}} .
$$

we see that the fermionic off-shell free energy reduces exactly to the regular boson off-shell free energy (A.14), establishing the duality of the CF and RB theories in their unHiggsed phases. The matching of off-shell free energies between the two theories automatically guarantees the matching of gap equations, as the latter are obtained by extremizing the off-shell free energies w.r.t. their 'fields'.

\section{A.3.2 Higgsed phase: $\operatorname{sgn}\left(X_{F}\right)=\operatorname{sgn}\left(\lambda_{B}\right)$}

In this case $\mathcal{F}\left(c_{F}, \zeta_{F}\right)$ simplifies to

$$
\begin{aligned}
\mathcal{F}\left(c_{F}, \zeta_{F}\right)= & \frac{N_{B}}{6 \pi}\left[-\frac{\lambda_{B}-2 \operatorname{sgn}\left(\lambda_{B}\right)}{\lambda_{B}} \hat{c}_{F}^{3}+\frac{3}{2 \lambda_{B}}\left(\frac{4 \pi \hat{\zeta}_{F}}{\kappa_{F}}\right)\left(\hat{c}_{F}^{2}-\hat{m}_{B}^{2}\right)+\right. \\
& +\frac{3 \hat{b}_{4}}{2 \lambda_{B}}\left(\frac{4 \pi \hat{\zeta}_{F}}{\kappa_{F}}\right)^{2}-\frac{\left(3 x_{6}^{B}+4\right)}{8 \lambda_{B}}\left(\frac{4 \pi \hat{\zeta}_{F}}{\kappa_{F}}\right)^{3}+ \\
& \left.+3 \int_{-\pi}^{\pi} d \alpha \rho_{B}(\alpha) \int_{\hat{c}_{F}}^{\infty} d y y\left(\log \left(1-e^{-y-i \alpha}\right)+\log \left(1-e^{-y+i \alpha}\right)\right)\right] .
\end{aligned}
$$

If we now make the field redefinitions

$$
\hat{c}_{F}=\hat{c}_{B}, \quad \frac{4 \pi \hat{\zeta}_{F}}{\kappa_{F}}=-2 \lambda_{B} \hat{\sigma}_{B},
$$

we find that (A.26) reduces to

$$
\begin{aligned}
F_{B}\left(c_{B}, \sigma_{B}\right)= & \frac{N_{B}}{6 \pi}\left[-\frac{\lambda_{B}-2 \operatorname{sgn}\left(\lambda_{B}\right)}{\lambda_{B}} \hat{c}_{B}^{3}-3 \hat{\sigma}_{B}\left(\hat{c}_{F}^{2}-\hat{m}_{B}^{2}\right)+6 \hat{b}_{4} \lambda_{B} \hat{\sigma}_{B}^{2}+\left(3 x_{6}^{B}+4\right) \lambda_{B}^{2} \hat{\sigma}_{B}^{3}\right. \\
& \left.\left.+3 \int_{-\pi}^{\pi} d \alpha \rho_{B}(\alpha) \int_{\hat{c}_{B}}^{\infty} d y y\left(\log \left(1-e^{-y-i \alpha}\right)+\log \left(1-e^{-y+i \alpha}\right)\right)\right] . \quad \text { (A. } 28\right)
\end{aligned}
$$

Eq. (A.28) may be regarded as the prediction of duality for the off-shell free energy of the RB theory in the Higgsed phase.

\section{B The tadpole from $W$ boson loops}

The exact all-orders propagator $G_{\mu \nu}(q)$ in (3.24) is the saddle point value in the large $N_{B}$ limit of a gauge-singlet field $\alpha_{\mu \nu}(q)$ that appears in [1] as one of two gauge-singlet fields $\alpha_{\mu \nu}$ and $\Sigma^{\mu \nu}$ that describe the effective dynamics of the $W$ boson:

$$
G_{\mu \nu}(q)=-\frac{1}{\lambda_{B}} \alpha_{\mu \nu}(q) .
$$


The first term of the gap equation (3.25) is then given by the tadpole contribution

$$
\lambda_{B} N_{B} T\left(\sigma_{B}\right)=\frac{\lambda_{B} N_{B}}{2 \pi} \int \frac{\mathcal{D}^{3} q}{(2 \pi)^{3}} g^{\mu \rho} G(q)_{\rho \mu}=-\frac{N_{B}}{2 \pi} \int \frac{\mathcal{D}^{3} q}{(2 \pi)^{3}} g^{\mu \rho} \alpha(q)_{\rho \mu} .
$$

For brevity, we work with the parameter $m=\lambda_{B} \sigma_{B}$ in what follows. The field $\alpha_{\mu \nu}(q)$ is given in terms of four known functions $F_{1}, F_{2}, F_{3}$ and $F_{4}$ of $w=2 q_{+} q_{-}$and the all-loop exact kernel $Q(q)=G^{-1}(q)$.

$$
\begin{aligned}
& \alpha_{++}(q)=\frac{\lambda_{B}}{(2 \pi)^{2} \operatorname{det} Q} \frac{1}{q_{-}^{2}}\left(i m F_{1}+\left(F_{3}+\frac{w}{2}\right)^{2}\right), \\
& \alpha_{-+}(q)=\frac{\lambda_{B}}{(2 \pi)^{2} \operatorname{det} Q}\left(\left(1-F_{4}\right)\left(F_{3}+\frac{w}{2}\right)-i m\left(F_{2}+i m-q_{3}\right)\right)=\alpha_{+-}(-q), \\
& \alpha_{--}(q)=\frac{\lambda_{B}}{(2 \pi)^{2} \operatorname{det} Q} q_{-}^{2}\left(1-F_{4}\right)^{2}, \\
& \alpha_{-3}(q)=-\frac{\lambda_{B}}{(2 \pi)^{2} \operatorname{det} Q} q_{-}\left(1-F_{4}\right)\left(F_{2}+i m-q_{3}\right)=\alpha_{3-}(-q), \\
& \alpha_{3+}(q)=-\frac{\lambda_{B}}{(2 \pi)^{2} \operatorname{det} Q} \frac{1}{q_{-}}\left(F_{1}\left(1-F_{4}\right)+\left(F_{2}+i m-q_{3}\right)\left(F_{3}+\frac{w}{2}\right)\right)=\alpha_{+3}(-q), \\
& \alpha_{33}(q)=-\frac{\lambda_{B}}{(2 \pi)^{2} \operatorname{det} Q}\left(\left(F_{2}+i m\right)^{2}-q_{3}^{2}\right) .
\end{aligned}
$$

We give explicit expressions for the functions $F_{1 \ldots 4}$ :

$$
\begin{aligned}
& F_{2}(w)=i m(g(w)-1), \quad F_{4}(w)=1-\frac{1}{g(w)} \\
& F_{3}(w)=-\frac{w}{2}+\frac{1}{g(w)}\left(\frac{1}{2} \mathcal{I}(w)-\frac{m^{2}}{3}\left(g(w)^{3}-g(0)^{3}\right)\right) \\
& F_{1}(w)=i m g(w)\left(c_{B}^{2}(g(w)-g(0))-\frac{m^{2}}{3}\left(g(w)^{3}-g(0)^{3}\right)+w g(w)-\mathcal{I}(w)\right),
\end{aligned}
$$

where the functions $g(w)$ and $\mathcal{I}(w)$ are given by

$$
g(w)=1+\lambda_{B} \xi(w), \quad \mathcal{I}(w)=\int_{0}^{w} d z g(z)=w+\lambda_{B} \mathcal{I}_{\xi}(w)
$$

and the function $\xi(w)$ in the definition of $g(w)$ above is given by

$$
\begin{aligned}
\xi(w)= & \frac{1}{2 m \beta} \int_{-\pi}^{\pi} d \alpha \rho_{B}(\alpha)\left[\log 2 \sinh \left(\frac{\beta}{2} \sqrt{w+c_{B}^{2}}+\frac{i}{2} \alpha\right)\right. \\
& \left.+\log 2 \sinh \left(\frac{\beta}{2} \sqrt{w+c_{B}^{2}}-\frac{i}{2} \alpha\right)\right] .
\end{aligned}
$$

We recognise the quantity $\mathcal{S}$ defined in (A.1) to be the value of $\beta m \xi(w)$ at $w=0$ :

$$
\mathcal{S}=\beta m \xi(0)=\frac{1}{2} \int_{-\pi}^{\pi} d \alpha \rho_{B}(\alpha)\left[\log 2 \sinh \left(\frac{\hat{c}_{B}+i \alpha}{2}\right)+\log 2 \sinh \left(\frac{\hat{c}_{B}-i \alpha}{2}\right)\right] .
$$


In the above expressions, the constant $c_{B}$ is the pole mass of the $W$ boson which occurs in the determinant of the all-loop kernel $Q(q)=G^{-1}(q)$ :

$$
\operatorname{det} Q(q)=-\frac{m}{(2 \pi)^{3}}\left(q^{2}+c_{B}^{2}\right),
$$

and is given in terms of the parameter $m$ and the function $\xi(w)$ above by

$$
c_{B}^{2}=m^{2}\left(1+\lambda_{B} \xi(0)\right)^{2} \quad \text { or equivalently } \quad \beta^{2} c_{B}^{2}=\hat{c}_{B}^{2}=\left(\hat{m}+\lambda_{B} \mathcal{S}\right)^{2} .
$$

Substituting the expressions (B.3), (B.4) for $\alpha_{\mu \nu}(q)$ in (B.2), we have

$$
\begin{aligned}
T(m) & =-\frac{1}{2 \pi \lambda_{B}} \int \frac{\mathcal{D}^{3} q}{(2 \pi)^{3}}\left(\alpha_{+-}(q)+\alpha_{-+}(q)+\alpha_{33}(q)\right) \\
& =\frac{1}{m} \int \frac{\mathcal{D}^{3} q}{(2 \pi)^{3}} \frac{1}{q^{2}+c_{B}^{2}}\left(\frac{\mathcal{I}(w)}{g(w)^{2}}+\frac{4 m^{2}}{3} g(w)+m^{2} g(w)^{2}+\frac{2 m^{2}}{3} \frac{g(0)^{3}}{g(w)^{2}}+q_{3}^{2}\right) \\
& =\frac{1}{m} \int \frac{\mathcal{D}^{3} q}{(2 \pi)^{3}} \frac{1}{q^{2}+c_{B}^{2}}\left(\mathcal{L}(w)-\left(w+c_{B}^{2}\right)\right)+\frac{1}{m} \int \frac{\mathcal{D}^{3} q}{(2 \pi)^{3}}
\end{aligned}
$$

where we have added and subtracted the term $w+c_{B}^{2}$ inside the integrand to complete the quantity $q_{3}^{2}$ to $q^{2}+c_{B}^{2}$. The quantity $\mathcal{L}(w)$ is given by

$$
\mathcal{L}(w)=\frac{\mathcal{I}(w)}{g(w)^{2}}+\frac{4}{3} m^{2} g(w)+m^{2} g(w)^{2}+\frac{2}{3} m^{2} \frac{g(0)^{3}}{g(w)^{2}} .
$$

The discrete sum over $q_{3}$ in the first term is given in terms of the function $\chi(w)$

$$
\begin{aligned}
\chi(w)= & -\frac{(2 \pi)^{3}}{m} \int \frac{\mathcal{D} q_{3}}{2 \pi} \frac{1}{q_{3}^{2}+w+c_{B}^{2}}=-\frac{(2 \pi)^{3}}{m \beta} \int_{-\pi}^{\pi} d \alpha \rho_{B}(\alpha) \sum_{n \in \mathbb{Z}} \frac{1}{\left(2 \pi \frac{n}{\beta}+\frac{\alpha}{\beta}\right)^{2}+w+c_{B}^{2}}, \\
= & -\frac{2 \pi^{3}}{m} \int_{-\pi}^{\pi} d \alpha \rho_{B}(\alpha) \frac{1}{\sqrt{w+c_{B}^{2}}} \times \\
& \times\left(\operatorname{coth}\left(\frac{\beta}{2} \sqrt{w+c_{B}^{2}}+\frac{i}{2} \alpha\right)+\operatorname{coth}\left(\frac{\beta}{2} \sqrt{w+c_{B}^{2}}-\frac{i}{2} \alpha\right)\right) .
\end{aligned}
$$

The $q_{3}$ sum in the last term in (B.10) is given by $c_{0} \equiv \sum_{n} 1$ and is hence divergent. We regularise the divergent sum using $\zeta$-function regularisation in which case we have $c_{0}$ (reg.) $=1+2 \zeta(0)=0$. Thus, equation (B.10) becomes

$$
T(m)=-\frac{1}{(2 \pi)^{3}} \int_{0}^{\infty} \frac{d w}{4 \pi} \chi(w)\left(\mathcal{L}(w)-\left(w+c_{B}^{2}\right)\right)=2 \int_{0}^{\infty} \frac{d w}{4 \pi} \xi^{\prime}(w)\left(\mathcal{L}(w)-\left(w+c_{B}^{2}\right)\right),
$$

where we have used $\chi(w)=-2(2 \pi)^{3} \xi^{\prime}(w)$. Next, recall the expressions (B.5) and (B.9):

$$
g(w)=1+\lambda_{B} \xi(w), \quad \mathcal{I}(w)=\int_{0}^{w} d z g(z)=w+\lambda_{B} \mathcal{I}_{\xi}(w), \quad c_{B}^{2}=m^{2}\left(1+\lambda_{B} \xi(0)\right)^{2} .
$$


Inserting (B.14) into (B.13) and Taylor-expanding $\mathcal{L}(w)-\left(w+c_{B}^{2}\right)$ in all explicit factors of $\lambda_{B}$ (around $\lambda_{B}=0$ ) we find

$$
\begin{aligned}
\mathcal{L}(w)-\left(w+c_{B}^{2}\right) & =\frac{\mathcal{I}(w)}{g(w)^{2}}+\frac{4}{3} m^{2} g(w)+m^{2} g(w)^{2}+\frac{2}{3} m^{2} \frac{g(0)^{3}}{g(w)^{2}}-\left(w+m^{2} g(0)^{2}\right) \\
& =\sum_{n=0}^{\infty}\left(-\lambda_{B}\right)^{n} \mathcal{L}_{n}(w)
\end{aligned}
$$

with

$$
\begin{aligned}
\mathcal{L}_{0}(w)= & 2 m^{2}, \quad \mathcal{L}_{1}(w)=-\left(\mathcal{I}_{\xi}(w)-2 w \xi(w)\right)-2 m^{2} \xi(w), \\
\mathcal{L}_{2}(w)= & \left(-2 \mathcal{I}_{\xi}(w) \xi(w)+3 w \xi(w)^{2}\right)+m^{2}\left(3 \xi(w)^{2}-4 \xi(w) \xi(0)+\xi(0)^{2}\right), \\
\mathcal{L}_{n}(w)= & \left((n+1) w \xi(w)^{n}-n \xi(w)^{n-1} \mathcal{I}_{\xi}(w)\right)+\frac{2 m^{2}}{3}\left((n+1) \xi(w)^{n}-(n-2) \xi(w)^{n-3} \xi(0)^{3}\right. \\
& \left.+3(n-1) \xi(w)^{n-2} \xi(0)^{2}-3 n \xi(w)^{n-1} \xi(0)\right) \quad \text { for } \quad n \geq 3 .
\end{aligned}
$$

The integral over $w$ in (B.13) becomes

$$
\sum_{n=0}^{\infty}\left(-\lambda_{B}\right)^{n} \int_{0}^{\infty} \frac{d w}{4 \pi} \xi^{\prime}(w) \mathcal{L}_{n}(w)
$$

The integral over the first two terms in the expressions for $\mathcal{L}_{n \geq 1}$ in (B.16) can be simplified by writing this in a total derivative form

$$
d w \xi^{\prime}(w)\left((n+1) \xi(w)^{n} w-n \xi(w)^{n-1} \mathcal{I}_{\xi}(w)\right)=d\left(\xi(w)^{n+1} w-\xi(w)^{n} \mathcal{I}_{\xi}(w)\right)
$$

In the dimensional regularisation scheme used in our previous paper [1] we have $\xi(\infty)=0$. Also, by definition we have $\mathcal{I}_{\xi}(0)=0$. This implies that

$$
\int_{0}^{\infty} d w \xi^{\prime}(w)\left((n+1) \xi(w)^{n} w-n \xi(w)^{n-1} \mathcal{I}_{\xi}(w)\right)=0 .
$$

The remaining terms in $\mathcal{L}$ are simple polynomials in $\xi$ and the integrations can be easily performed. Only $\mathcal{L}_{0}$ and $\mathcal{L}_{1}$ give non-zero contributions:

$$
\int_{0}^{\infty} d w \xi^{\prime}(w) \mathcal{L}_{0}(w)=-2 m^{2} \xi(0), \quad \int_{0}^{\infty} d w \xi^{\prime}(w) \mathcal{L}_{1}(w)=+m^{2} \xi(0)^{2} .
$$

Substituting the above results into (B.13), we get

$$
T(m)=-\frac{m^{2}}{2 \pi}\left(2 \xi(0)+\lambda_{B} \xi(0)^{2}\right) .
$$

Recalling the equation (B.9) for $\hat{c}_{B}$ and $m=\lambda_{B} \sigma_{B}$, we have the final expression for the tadpole contribution from the $W$ boson propagator (B.2):

$$
\lambda_{B} N_{B} T\left(\sigma_{B}\right)=-\frac{N_{B}}{2 \pi}\left(c_{B}^{2}-\lambda_{B}^{2} \sigma_{B}^{2}\right)
$$




\section{The critical boson scaling limit}

Recall that the RB theory reduces to the critical boson or CB theory in the scaling limit

$$
m_{B}^{2} \rightarrow \infty, \quad \lambda_{B} b_{4} \rightarrow \infty, \quad \frac{m_{B}^{2}}{2 \lambda_{B} b_{4}}=m_{B}^{\text {cri }}=\text { fixed } .
$$

In this subsection we study the reduction of the off-shell free energy under this scaling limit. The off-shell free energy (4.2) simplifies in the limit (C.1) as follows. The second term in the second line of (4.2) reduces to

$$
6 \lambda_{B} \hat{b}_{4} \hat{\sigma}_{B}\left(\hat{m}_{B}^{\text {cri }}+\hat{\sigma}_{B}\right)=6 \lambda_{B} \hat{b}_{4}\left[\left(\hat{\sigma}_{B}+\frac{\hat{m}_{B}^{\text {cri }}}{2}\right)^{2}-\frac{\left(\hat{m}_{B}^{\text {cri }}\right)^{2}}{4}\right]
$$

Note that confining potential (C.2) is infinitely stiff in the CB scaling limit. It follows that $\hat{\sigma}_{B}$ is frozen at the minimum of (C.2) i.e. at $\sigma_{B}=-\frac{m_{B}^{\text {cri }}}{2}$ in the CB scaling limit. It follows that in this limit (4.2) simplifies to

$$
\begin{aligned}
F\left(c_{B}, \tilde{\mathcal{S}}\right)= & \frac{N_{B}}{6 \pi}\left[-\hat{c}_{B}^{3}-4 \tilde{\mathcal{S}}^{3} \lambda_{B}^{2}+\frac{3}{2} \hat{c}_{B}^{2} \hat{m}_{B}^{\text {cri }}\right. \\
& +6 \tilde{\mathcal{S}}^{2} \lambda_{B}^{2} \hat{m}_{B}^{\text {cri }}-3 \tilde{\mathcal{S}} \lambda_{B}^{2}\left(\hat{m}_{B}^{\text {cri }}\right)^{2}+6 \hat{c}_{B}\left|\lambda_{B}\right|\left(\tilde{\mathcal{S}}-\frac{\hat{m}_{B}^{\text {cri }}}{2}\right)^{2} \\
& \left.+3 \int_{-\pi}^{\pi} d \alpha \rho_{B}(\alpha) \int_{\hat{c}_{B}}^{\infty} d y y\left(\log \left(1-e^{-y-i \alpha}\right)+\log \left(1-e^{-y+i \alpha}\right)\right)\right] .
\end{aligned}
$$

(we have omitted a divergent constant proportional to $b_{4}$ that can be cancelled by a cosmological constant counterterm.) Extremizing (C.3) w.r.t. $\tilde{\mathcal{S}}$ we recover the first of the gap equations in (4.3) under the replacement

$$
\sigma_{B} \rightarrow-\frac{m_{B}^{\text {cri }}}{2}
$$

The two inequivalent solutions of this equation are (4.4) and (4.5) under the same replacement for $\sigma_{B}$. These solutions correspond to the unHiggsed and Higgsed branches respectively.

On the Higgsed branch we can plug the solution of (4.5) back into (C.3) to find a free energy as a function of the single off-shell variable $c_{B}$; the final result of this exercise is given by the critical boson free energy given in (3.13). ${ }^{24}$ In a similar manner, on the unHiggsed branch we can plug the solution of (4.4) into (C.3) to find off-shell free energy as a function of $c_{B}$, given by

$$
\begin{aligned}
F_{\mathrm{CB}}\left(c_{B}\right)= & \frac{N_{B}}{6 \pi}\left[-\hat{c}_{B}^{3}+\frac{3}{2} \hat{m}_{B}^{\mathrm{cri}} \hat{c}_{B}^{2}-\frac{\lambda_{B}^{2}\left(\hat{m}_{B}^{\mathrm{cri}}\right)^{3}}{2}\right. \\
& \left.+3 \int_{-\pi}^{\pi} \rho(\alpha) d \alpha \int_{\hat{c}_{B}}^{\infty} d y y\left(\log \left(1-e^{-y-i \alpha}\right)+\log \left(1-e^{-y+i \alpha}\right)\right)\right] .
\end{aligned}
$$

\footnotetext{
${ }^{24}$ This procedure automatically produces a particular choice of the cosmological constant counterterm. It would be interesting to investigate if this particular value has physical significance.
} 
Open Access. This article is distributed under the terms of the Creative Commons Attribution License (CC-BY 4.0), which permits any use, distribution and reproduction in any medium, provided the original author(s) and source are credited.

\section{References}

[1] S. Choudhury et al., Bose-Fermi Chern-Simons Dualities in the Higgsed Phase, arXiv: 1804.08635 [INSPIRE].

[2] S. Minwalla and S. Yokoyama, Chern Simons Bosonization along RG Flows, JHEP 02 (2016) 103 [arXiv:1507.04546] [INSPIRE].

[3] O. Aharony, S. Jain and S. Minwalla, Flows, Fixed Points and Duality in Chern-Simons-matter theories, arXiv:1808.03317 [INSPIRE].

[4] O. Aharony, G. Gur-Ari and R. Yacoby, Correlation Functions of Large N Chern-Simons-Matter Theories and Bosonization in Three Dimensions, JHEP 12 (2012) 028 [arXiv: 1207.4593] [INSPIRE].

[5] S. Jain, M. Mandlik, S. Minwalla, T. Takimi, S.R. Wadia and S. Yokoyama, Unitarity, Crossing Symmetry and Duality of the S-matrix in large N Chern-Simons theories with fundamental matter, JHEP 04 (2015) 129 [arXiv:1404.6373] [INSPIRE].

[6] S. Yokoyama, Scattering Amplitude and Bosonization Duality in General Chern-Simons Vector Models, JHEP 09 (2016) 105 [arXiv: 1604.01897] [INSPIRE].

[7] S. Jain, S. Minwalla, T. Sharma, T. Takimi, S.R. Wadia and S. Yokoyama, Phases of large N vector Chern-Simons theories on $S^{2} \times S^{1}$, JHEP 09 (2013) 009 [arXiv:1301.6169] [INSPIRE].

[8] S. Giombi, V. Kirilin and E. Skvortsov, Notes on Spinning Operators in Fermionic CFT, JHEP 05 (2017) 041 [arXiv: 1701.06997] [INSPIRE].

[9] Z. Komargodski and N. Seiberg, A symmetry breaking scenario for $Q C D_{3}$, JHEP 01 (2018) 109 [arXiv: 1706.08755] [INSPIRE].

[10] E. Sezgin, E.D. Skvortsov and Y. Zhu, Chern-Simons Matter Theories and Higher Spin Gravity, JHEP 07 (2017) 133 [arXiv:1705. 03197] [INSPIRE].

[11] K. Aitken, A. Baumgartner, A. Karch and B. Robinson, 3d Abelian Dualities with Boundaries, JHEP 03 (2018) 053 [arXiv:1712.02801] [INSPIRE].

[12] A. Karch, D. Tong and C. Turner, Mirror Symmetry and Bosonization in 2d and 3d, JHEP 07 (2018) 059 [arXiv: 1805.00941] [INSPIRE].

[13] O. Aharony, L.F. Alday, A. Bissi and R. Yacoby, The Analytic Bootstrap for Large N Chern-Simons Vector Models, JHEP 08 (2018) 166 [arXiv:1805.04377] [INSPIRE].

[14] R. Yacoby, Scalar Correlators in Bosonic Chern-Simons Vector Models, arXiv:1805.11627 [INSPIRE].

[15] K. Aitken, A. Baumgartner and A. Karch, Novel 3d bosonic dualities from bosonization and holography, JHEP 09 (2018) 003 [arXiv: 1807.01321] [INSPIRE].

[16] O. Aharony, J. Marsano, S. Minwalla, K. Papadodimas and M. Van Raamsdonk, The Hagedorn - deconfinement phase transition in weakly coupled large $N$ gauge theories, Adv. Theor. Math. Phys. 8 (2004) 603 [hep-th/0310285] [InSPIRE]. 
[17] I.R. Klebanov and A.M. Polyakov, AdS dual of the critical $O(N)$ vector model, Phys. Lett. B 550 (2002) 213 [hep-th/0210114] [INSPIRE].

[18] E. Sezgin and P. Sundell, Massless higher spins and holography, Nucl. Phys. B 644 (2002) 303 [Erratum ibid. B 660 (2003) 403] [hep-th/0205131] [INSPIRE].

[19] S. Giombi and X. Yin, Higher Spin Gauge Theory and Holography: The Three-Point Functions, JHEP 09 (2010) 115 [arXiv:0912.3462] [INSPIRE].

[20] S. Giombi, S. Minwalla, S. Prakash, S.P. Trivedi, S.R. Wadia and X. Yin, Chern-Simons Theory with Vector Fermion Matter, Eur. Phys. J. C 72 (2012) 2112 [arXiv:1110.4386] [INSPIRE].

[21] C.-M. Chang, S. Minwalla, T. Sharma and X. Yin, ABJ Triality: from Higher Spin Fields to Strings, J. Phys. A 46 (2013) 214009 [arXiv:1207.4485] [InSPIRE].

[22] J. Maldacena and A. Zhiboedov, Constraining Conformal Field Theories with A Higher Spin Symmetry, J. Phys. A 46 (2013) 214011 [arXiv:1112.1016] [InSPIRE].

[23] J. Maldacena and A. Zhiboedov, Constraining conformal field theories with a slightly broken higher spin symmetry, Class. Quant. Grav. 30 (2013) 104003 [arXiv:1204.3882] [INSPIRE].

[24] G. Gur-Ari and R. Yacoby, Correlators of Large N Fermionic Chern-Simons Vector Models, JHEP 02 (2013) 150 [arXiv:1211.1866] [INSPIRE].

[25] A. Bedhotiya and S. Prakash, A test of bosonization at the level of four-point functions in Chern-Simons vector models, JHEP 12 (2015) 032 [arXiv:1506.05412] [INSPIRE].

[26] G.J. Turiaci and A. Zhiboedov, Veneziano Amplitude of Vasiliev Theory, JHEP 10 (2018) 034 [arXiv: 1802.04390] [INSPIRE].

[27] N. Seiberg, T. Senthil, C. Wang and E. Witten, A Duality Web in $2+1$ Dimensions and Condensed Matter Physics, Annals Phys. 374 (2016) 395 [arXiv:1606.01989] [INSPIRE].

[28] J. Murugan and H. Nastase, Particle-vortex duality in topological insulators and superconductors, JHEP 05 (2017) 159 [arXiv:1606.01912] [INSPIRE].

[29] S. Yokoyama, Chern-Simons-Fermion Vector Model with Chemical Potential, JHEP 01 (2013) 052 [arXiv: 1210.4109] [InSPIRE].

[30] S. Jain, S.P. Trivedi, S.R. Wadia and S. Yokoyama, Supersymmetric Chern-Simons Theories with Vector Matter, JHEP 10 (2012) 194 [arXiv: 1207.4750] [INSPIRE].

[31] O. Aharony, S. Giombi, G. Gur-Ari, J. Maldacena and R. Yacoby, The Thermal Free Energy in Large N Chern-Simons-Matter Theories, JHEP 03 (2013) 121 [arXiv:1211.4843] [INSPIRE].

[32] T. Takimi, Duality and higher temperature phases of large $N$ Chern-Simons matter theories on $S^{2} \times S^{1}$, JHEP 07 (2013) 177 [arXiv:1304.3725] [INSPIRE].

[33] S. Yokoyama, A Note on Large $N$ Thermal Free Energy in Supersymmetric Chern-Simons Vector Models, JHEP 01 (2014) 148 [arXiv:1310.0902] [INSPIRE].

[34] S. Jain, S. Minwalla and S. Yokoyama, Chern Simons duality with a fundamental boson and fermion, JHEP 11 (2013) 037 [arXiv:1305.7235] [INSPIRE].

[35] G. Gur-Ari and R. Yacoby, Three Dimensional Bosonization From Supersymmetry, JHEP 11 (2015) 013 [arXiv: 1507.04378] [INSPIRE]. 
[36] M. Geracie, M. Goykhman and D.T. Son, Dense Chern-Simons Matter with Fermions at Large N, JHEP 04 (2016) 103 [arXiv: 1511.04772] [INSPIRE].

[37] K. Inbasekar, S. Jain, S. Mazumdar, S. Minwalla, V. Umesh and S. Yokoyama, Unitarity, crossing symmetry and duality in the scattering of $\mathcal{N}=1$ SUSY matter Chern-Simons theories, JHEP 10 (2015) 176 [arXiv:1505.06571] [INSPIRE].

[38] Y. Dandekar, M. Mandlik and S. Minwalla, Poles in the S-Matrix of Relativistic Chern-Simons Matter theories from Quantum Mechanics, JHEP 04 (2015) 102 [arXiv: 1407.1322] [INSPIRE].

[39] K. Inbasekar, S. Jain, P. Nayak and V. Umesh, All tree level scattering amplitudes in Chern-Simons theories with fundamental matter, Phys. Rev. Lett. 121 (2018) 161601 [arXiv: 1710.04227] [INSPIRE].

[40] K. Inbasekar et al., Dual Superconformal Symmetry of $\mathcal{N}=2$ Chern-Simons theory with Fundamental Matter and Non-Renormalization at Large N, arXiv:1711.02672 [INSPIRE].

[41] F. Benini, C. Closset and S. Cremonesi, Comments on 3d Seiberg-like dualities, JHEP 10 (2011) 075 [arXiv: 1108.5373] [INSPIRE].

[42] G. Gur-Ari, S.A. Hartnoll and R. Mahajan, Transport in Chern-Simons-Matter Theories, JHEP 07 (2016) 090 [arXiv:1605.01122] [INSPIRE].

[43] O. Aharony, G. Gur-Ari and R. Yacoby, $d=3$ Bosonic Vector Models Coupled to Chern-Simons Gauge Theories, JHEP 03 (2012) 037 [arXiv:1110.4382] [INSPIRE].

[44] T. Nosaka and S. Yokoyama, Complete factorization in minimal $\mathcal{N}=4$ Chern-Simons-matter theory, JHEP 01 (2018) 001 [arXiv:1706.07234] [INSPIRE].

[45] S. Giombi, Higher Spin - CFT Duality, in Proceedings, Theoretical Advanced Study Institute in Elementary Particle Physics: New Frontiers in Fields and Strings (TASI 2015): Boulder, CO, USA, June 1-26, 2015, pp. 137-214, 2017, arXiv:1607.02967, DOI [INSPIRE].

[46] S.R. Wadia, Chern-Simons theories with fundamental matter: A brief review of large $N$ results including Fermi-Bose duality and the S-matrix, Int. J. Mod. Phys. A 31 (2016) 1630052 .

[47] Y. Frishman and J. Sonnenschein, Large N Chern-Simons with massive fundamental fermions - A model with no bound states, JHEP 12 (2014) 165 [arXiv:1409.6083] [INSPIRE].

[48] V. Gurucharan and S. Prakash, Anomalous dimensions in non-supersymmetric bifundamental Chern-Simons theories, JHEP 09 (2014) 009 [Erratum ibid. 1711 (2017) 045] [arXiv: 1404.7849] [INSPIRE].

[49] S. Giombi, Testing the Boson/Fermion Duality on the Three-Sphere, arXiv:1707.06604 [INSPIRE].

[50] W.A. Bardeen, The Massive Fermion Phase for the U(N) Chern-Simons Gauge Theory in $D=3$ at Large $N$, JHEP 10 (2014) 39 [arXiv:1404.7477] [INSPIRE].

[51] W.A. Bardeen and M. Moshe, Spontaneous breaking of scale invariance in a $D=3 \mathrm{U}(N)$ model with Chern-Simons gauge fields, JHEP 06 (2014) 113 [arXiv:1402.4196] [INSPIRE].

[52] M. Moshe and J. Zinn-Justin, 3D Field Theories with Chern-Simons Term for Large $N$ in the Weyl Gauge, JHEP 01 (2015) 054 [arXiv:1410.0558] [INSPIRE]. 
[53] S. Giombi, V. Gurucharan, V. Kirilin, S. Prakash and E. Skvortsov, On the Higher-Spin Spectrum in Large $N$ Chern-Simons Vector Models, JHEP 01 (2017) 058 [arXiv: 1610.08472] [INSPIRE].

[54] V. Guru Charan and S. Prakash, On the Higher Spin Spectrum of Chern-Simons Theory coupled to Fermions in the Large Flavour Limit, JHEP 02 (2018) 094 [arXiv:1711.11300] [INSPIRE].

[55] D. Radičević, Disorder Operators in Chern-Simons-Fermion Theories, JHEP 03 (2016) 131 [arXiv: 1511.01902] [INSPIRE].

[56] O. Aharony, Baryons, monopoles and dualities in Chern-Simons-matter theories, JHEP 02 (2016) 093 [arXiv: 1512.00161] [INSPIRE].

[57] O. Aharony, P. Narayan and T. Sharma, On monopole operators in supersymmetric Chern-Simons-matter theories, JHEP 05 (2015) 117 [arXiv: 1502.00945] [INSPIRE].

[58] D. Radičević, D. Tong and C. Turner, Non-Abelian 3d Bosonization and Quantum Hall States, JHEP 12 (2016) 067 [arXiv: 1608.04732] [INSPIRE].

[59] O. Aharony, F. Benini, P.-S. Hsin and N. Seiberg, Chern-Simons-matter dualities with SO and USp gauge groups, JHEP 02 (2017) 072 [arXiv: 1611.07874] [INSPIRE].

[60] A. Karch and D. Tong, Particle-Vortex Duality from 3d Bosonization, Phys. Rev. X 6 (2016) 031043 [arXiv: 1606.01893] [INSPIRE].

[61] A. Karch, B. Robinson and D. Tong, More Abelian Dualities in $2+1$ Dimensions, JHEP 01 (2017) 017 [arXiv: 1609.04012] [INSPIRE].

[62] P.-S. Hsin and N. Seiberg, Level/rank Duality and Chern-Simons-Matter Theories, JHEP 09 (2016) 095 [arXiv : 1607.07457] [INSPIRE].

[63] J. Gomis, Z. Komargodski and N. Seiberg, Phases Of Adjoint QCD $D_{3}$ And Dualities, SciPost Phys. 5 (2018) 007 [arXiv: 1710.03258] [InSPIRE].

[64] K. Jensen, A master bosonization duality, JHEP 01 (2018) 031 [arXiv:1712.04933] [INSPIRE].

[65] D. Gaiotto, Z. Komargodski and N. Seiberg, Time-reversal breaking in $Q C D_{4}$, walls and dualities in $2+1$ dimensions, JHEP 01 (2018) 110 [arXiv:1708.06806] [INSPIRE].

[66] F. Benini, P.-S. Hsin and N. Seiberg, Comments on global symmetries, anomalies and duality in $(2+1) d$, JHEP 04 (2017) 135 [arXiv:1702.07035] [INSPIRE].

[67] K. Jensen and A. Karch, Embedding three-dimensional bosonization dualities into string theory, JHEP 12 (2017) 031 [arXiv:1709.07872] [INSPIRE].

[68] K. Jensen and A. Karch, Bosonizing three-dimensional quiver gauge theories, JHEP 11 (2017) 018 [arXiv: 1709. 01083] [inSPIRE].

[69] F. Benini, Three-dimensional dualities with bosons and fermions, JHEP 02 (2018) 068 [arXiv:1712.00020] [INSPIRE].

[70] C. Cordova, P.-S. Hsin and N. Seiberg, Global Symmetries, Counterterms and Duality in Chern-Simons Matter Theories with Orthogonal Gauge Groups, SciPost Phys. 4 (2018) 021 [arXiv: 1711.10008] [INSPIRE]. 